\title{
Analytic Torsion and Holomorphic Determinant Bundles I. Bott-Chern Forms and Analytic Torsion
}

\author{
J.-M. Bismut ${ }^{1}$, H. Gillet ${ }^{2}$ and C. Soulé ${ }^{3}$ \\ Département de Mathématique, Université Paris-Sud, Bât. 425, F-91405 Orsay Cedex, France \\ Department of Mathematics, University of Illinois at Chicago, Chicago, IL 60638, USA \\ 3 CNRS LA212 and I.H.E.S., 35 route de Chartres, F-91440 Bures-Sur-Yvette, France
}

\begin{abstract}
We attach secondary invariants to any acyclic complex of holomorphic Hermitian vector bundles on a complex manifold. These were first introduced by Bott and Chern [Bot C]. Our new definition uses Quillen's superconnections. We also give an axiomatic characterization of these classes. These results will be used in [BGS2] and [BGS3] to study the determinant of the cohomology of a holomorphic vector bundle.
\end{abstract}

\section{Contents}

Introduction. . . . . . . . . . . . . . . . . . . . . 49

a) Determinant of a Chain Complex of Vector Spaces: . . . . . . . 52

1. Torsion for an Acyclic Chain Complex . . . . . . . . . . 52

2. Determinant and Cohomology of a Chain Complex. . . . . . 54

3. Torsion and Analytic Torsion of a Hermitian Chain Complex . . 55

b) Number Operator and the Chern Character Forms of a Holomorphic Hermitian Chain Complex . . . . . . . . . . . . . . 56

c) Double Transgression of the Chern Character Forms . . . . . . 63

d) Multiplicativity Property of the Generalized Analytic Torsion . . . 65

e) Bott-Chern Classes . . . . . . . . . . . . . . . . . . . . . . . 69

f) Axiomatic Definition of the Secondary Classes of Bott and Chern . . 74

\section{Introduction}

This is the first of a series of three papers, which are devoted to the study of the determinant line bundle of the direct image of a holomorphic vector bundle. Parts II and III of this work will be referred to as [BGS2] and [BGS3]. We first summarize the results which are obtained in these papers.

Let $\pi: M \rightarrow B$ be a proper holomorphic map of complex analytic manifolds and let $\xi$ be a complex holomorphic vector bundle on $M$.

According to Grothendieck and Knudsen-Mumford [KM], the (derived) direct 
image of $\xi$ by $\pi$ has a determinant, which is a holomorphic line bundle $\operatorname{det} R \pi_{*} \xi$ on $B$. We call its inverse $\lambda^{\mathrm{KM}}=\left(\operatorname{det} R \pi_{*} \xi\right)^{-1}$ the Knudsen-Mumford determinant.

For every $y \in B$, let $Z_{y}=\pi^{-1}\{y\}$ be the fiber over $y$, and for $i \geqq 0$, let $H^{i}\left(Z_{y}, \xi\right)$ be the $i^{\text {th }}$ cohomology of $Z_{y}$ with coefficients in the restriction of $\xi$ to $Z_{y}$. The fiber $\lambda_{y}^{\mathrm{KM}}$ is by definition given by

$$
\lambda_{y}^{\mathrm{KM}}=\bigotimes_{l \geqq 0} \operatorname{det} H^{i}\left(Z_{y}, \xi\right)^{(-1)^{i+1}} .
$$

Assume now that for every $y \in B$, there is a Kähler metric $g^{Z_{y}}$ on $Z_{y}$ depending smoothly on $y \in B$. The holomorphic tangent bundle $T^{(1,0)} Z$ is then endowed with a Hermitian metric $g^{Z}$. Assume furthermore that $\xi$ is endowed with a Hermitian metric $h^{\xi}$. If $l=\operatorname{dim} Z_{y}$, let

$$
0 \rightarrow E_{y}^{0} \underset{\overline{\delta_{y}}}{\longrightarrow} E_{y}^{1} \rightarrow \cdots \rightarrow E_{y}^{l} \rightarrow 0
$$

be the $\bar{\partial}$ complex associated with the restriction of $\xi$ to $Z_{y}$.

In [Q2], Quillen suggested that the fibers $\lambda_{y}^{\mathrm{KM}}$ can be naturally endowed with a metric, which is the product of the $L^{2}$ metric, deduced from integration along the fiber, by the analytic torsion of Ray-Singer [RS]. He considers the situation where $M$ is the family of Cauchy-Riemann operators on a fixed Hermitian smooth vector bundle over a Riemann surface. Quillen constructs a smooth line bundle $\lambda$ on $B$ which has the following three properties:

- For every $y \in B, \lambda_{y}$ is canonically isomorphic to $\lambda_{y}^{\mathrm{KM}}$.

$-\lambda_{y}$ has a natural holomorphic structure.

-When endowed with the Quillen metric, the curvature of the canonical holomorphic Hermitian connection on $\lambda$ is obtained by a differential form version of the Riemann-Roch-Grothendieck Theorem.

In $[\mathrm{BF} 1,2]$, Bismut and Freed considered the case of $C^{\infty}$ fibrations $M \underset{\pi}{\longrightarrow} B$.

They constructed a $C^{\infty}$ line bundle $\lambda$ on $B$ associated with a family of Dirac operators. In $[\mathrm{BF} 1,2]$, the line bundle $\lambda$ was endowed with a metric and with a unitary connection. The curvature of this connection was shown to be given by a differential form version of the Atiyah-Singer Index Theorem for families [AS]. Finally, it was proved in [BF1] that in the case considered by Quillen [Q2], the constructions of [BF1] and of [Q2] coincide.

In the case where $M$ and $B$ are complex manifolds and $\pi$ is holomorphic, an application of the results of Bismut and Freed [BF1,2] tells us that there exists a connection ${ }^{1} \nabla$ on $\lambda$ which is unitary for the Quillen metric, and whose curvature is of type $(1,1)$. Therefore by [AHS, Theorem 5.1], we know that $\lambda$ can be endowed with a holomorphic structure such that ${ }^{1} \nabla$ is the corresponding unique holomorphic Hermitian connection.

Now observe that for every $y \in B, \lambda_{y}^{\mathrm{KM}}$ and $\lambda_{y}$ are canonically isomorphic. However it is not at all clear that the isomorphism of the fibers extends into a holomorphic, or even a $C^{\infty}$ isomorphism.

We prove in full generality that this isomorphism is smooth and that it holomorphic when $\pi$ is locally Kähler, i.e. there is an open covering $\mathscr{U}$ of $B$ such that, if $U \in \mathscr{U}, \pi^{-1}(U)$ admits a Kähler metric (whose restriction to $Z_{y}, y \in U$, may differ from $g^{Z_{y}}$ ). 
Let $R^{Z}, L^{\xi}$ be the curvatures of the holomorphic Hermitian connections on $T^{(1,0)} Z$ and $\xi$.

Theorem 0.1. Assume that $\pi$ is locally Kähler. Then the identification of the fibers $\lambda_{y} \simeq \lambda_{y}^{\mathrm{KM}}$ defines a holomorphic isomorphism of line bundles $\lambda \simeq \lambda^{\mathrm{KM}}$. The curvature of the holomorphic connection associated with the Quillen metric on $\lambda \simeq \lambda^{\mathrm{KM}}$ is the component of degree 2 in the following form on $B$,

$$
2 i \pi \int_{Z} \operatorname{Td}\left(-R^{Z} / 2 i \pi\right) \operatorname{Tr}\left[\exp \left(-L^{\xi} / 2 i \pi\right)\right] .
$$

Let now $g^{\prime Z}$ be another choice of a Kähler metric in the fibers $Z$, with associated curvature $R^{\prime Z}$. Bott and Chern [Bot C, 3.28] defined a class of forms $\widetilde{T d}\left(g^{Z}, g^{\prime Z}\right)$ (modulo the images of $\partial^{M}$ and $\bar{\partial}^{M}$ ) such that

$$
(1 / 2 i \pi) \bar{\partial}^{M} \partial^{M} \widetilde{T d}\left(g^{Z}, g^{Z}\right)=T d\left(-R^{\prime Z} / 2 i \pi\right)-T d\left(-R^{Z} / 2 i \pi\right) .
$$

Theorem 0.2. If $g^{Z}$ is replaced $g^{\prime Z}$, the Quillen metric on $\lambda^{\mathrm{KM}}$ is multiplied by the exponential of the component of degree 0 in

$$
\int_{Z} \widetilde{T d}\left(g^{Z}, g^{\prime}\right) \operatorname{Tr}\left[\exp \left(-L^{\xi} / 2 i \pi\right)\right]
$$

Finally, let

$$
0 \rightarrow \xi_{0} \underset{v}{\longrightarrow} \xi_{1} \underset{v}{\longrightarrow} \cdots \underset{v}{\longrightarrow} \xi_{m} \rightarrow 0
$$

be an acyclic complex of holomorphic bundles, equipped with Hermitian metrics. Let $\lambda_{j}^{\mathrm{KM}}$ be the Knudsen-Mumford determinant of $\xi_{j}, j \geqq 0$. According to [KM], the line bundle $\bigotimes_{J \geq 0}\left(\lambda_{j}^{\mathrm{KM}}\right)^{(-1)^{j}}$ is canonically trivial. Let $\sigma$ be the canonical section of this line bundle.

On the other hand, we define in this first paper a class of forms $\widetilde{c h}(\xi)$ on $M$ such that

$$
(1 / 2 i \pi) \bar{\partial}^{M} \partial^{M} \tilde{\operatorname{ch}}(\xi)=\sum_{j \geqq 0}(-1)^{j+1} \operatorname{Tr}\left[\exp \left(-L^{\xi_{j}} / 2 i \pi\right)\right] .
$$

Theorem 0.3. The Quillen norm of $\sigma$ is the exponential of the component of degree 0 in

$$
-\frac{1}{2} \int_{Z} T d\left(-R^{Z} / 2 i \pi\right) \widetilde{\operatorname{ch}}(\xi)
$$

Also, we obtain several results on characteristic classes for direct images in degree higher than 0 and 2. In particular, an analog of Theorem 0.3 is proved in [BGS2] in any degree, and is related to work by Gillet and Soule $[\mathrm{GS} 1,2]$ on direct images in Arakelov theory.

When the fibers are curves, some of our results were already known before. In [BeK], Belavin and Knizhnik obtained Theorem 0.1 in relative dimension 1 for specific line bundles. A proof of Theorem 0.1 in relative dimension 1 was announced by Bost [Bo] and Freed [F].

Also Donaldson [D2] has used the results of $[\mathrm{BF} 1,2]$ to extend the results of Quillen [Q2] in relative dimension higher than one. When $M=Z \times B$, and when $\xi$ has no higher dimensional cohomology, a proof of Theorem 0.1 was announced by Gillet and Soulé [GS2]. 
In the case where the fibers $Z$ are curves, Theorem 0.2 is a well-known result. Polyakov's computation of the conformal anomaly $[\mathrm{P}]$ can be seen as an example of Theorem 0.2 , as explained in [Al], [Bo].

Let us now describe the content of this first article. Its purpose is to study secondary invariants attached to acyclic complexes of holomorphic Hermitian vector bundles on a complex manifold. Such invariants have been introduced by Bott and Chern [Bot C] and studied by Donaldson [D1]. Also Gillet and Soulé gave in [GS2] another definition of these Bott-Chern forms.

The Bott-Chern forms are holomorphic analogs of those of Chern-Simons in differential geometry. These are obtained by transgression from the Chern character forms. But, in the holomorphic context, instead of writing an exact form $\alpha$ as $\alpha=d \beta$, one has to solve $\alpha=\partial \bar{\partial} \gamma$, and give explicit formulas for $\gamma$. Such a double transgression was first achieved in [Bot C].

In [Q1], Quillen introduced superconnections on $\mathbb{Z}_{2}$ graded finite dimensional vector bundles, to obtain non-trivial representatives of the Chern character of difference bundles. Also the analogs of Chern-Simons forms were introduced in [Q1] for superconnections to transgress the Chern character forms.

Here we use Quillen's superconnections to construct new representatives of Bott-Chern classes of an acyclic complex. Our formula in Theorem 1.17 uses in a crucial way the number operator, which describes the $\mathbb{Z}$-grading of the complex.

We also establish certain identities which will be very useful in [BGS2] when computing certain asymptotic expansions. After giving a new proof of one of Bott and Chern's results [Bot C] in Theorem 1.27, we conclude with a third (and axiomatic) construction of their characteristic classes in Theorem 1.29, along the lines of [GS2].

This paper is organized as follows.

Section a) contains preliminaries on the determinant of a complex of vector spaces.

In b), we establish algebraic identities on the Chern forms associated to a holomorphic acyclic chain complex.

In c), we calculate the double transgression of such Chern forms by using higher order analytic torsion forms.

In d), we establish how such forms behave when considering double complexes and exact sequences.

In e), we construct the classes of Bott-Chern [Bot $\mathrm{C}$ ] and we reprove a result of Donaldson [D1].

In $\mathrm{f}$ ), we give an axiomatic construction of the double transgression of the characteristic classes associated with a chain complex. The classes which were obtained in the previous sections are shown to verify these axioms.

The results contained in our three papers were announced in [BGS1].

\section{a) Determinant of a Chain Complex of Vector Spaces}

1. Torsion of an Acyclic Chain Complex. If $E$ is a finite dimensional complex vector space of dimension $n$, set

$$
\operatorname{det} E=\Lambda^{n}(E)
$$


Let

$$
(E, \bar{\partial}): 0 \rightarrow E^{0} \underset{\bar{\partial}}{\longrightarrow} E^{1} \underset{\bar{\partial}}{\longrightarrow} \cdots \rightarrow E^{l} \rightarrow 0
$$

be a chain complex of finite dimensional complex vector spaces, with chain map $\bar{\partial}$ (and so $\bar{\partial}^{2}=0$ ). The determinant of $E$ is the one dimensional complex vector space

$$
\lambda=\operatorname{det}(E)=\left(\operatorname{det} E^{0}\right)^{-1} \otimes \operatorname{det} E^{1} \otimes\left(\operatorname{det} E^{2}\right)^{-1} \otimes \cdots .
$$

Assume that $(E, \bar{\partial})$ is acyclic. For $0 \leqq i \leqq l-1 \operatorname{set} n_{i}=\operatorname{dim}\left[\bar{\partial}\left(E^{i}\right)\right]$. Let $s_{i} \in \Lambda^{n_{i}}\left(E^{i}\right)$ be such that $\bar{\partial} s_{i} \neq 0$. Since $(E, \bar{\partial})$ is acyclic, $\bar{\partial} s_{i} \wedge s_{i+1}$ is non-zero in $\operatorname{det}\left(E^{i+1}\right)$.

Definition 1.1. $T(\bar{\partial})$ is the non-zero element in $\operatorname{det}(E)$,

$$
T(\bar{\partial})=\left(s^{0}\right)^{-1} \otimes \bar{\partial} s^{0} \wedge s^{1} \otimes\left(\bar{\partial} s^{1} \wedge s^{2}\right)^{-1} \otimes \cdots
$$

Of course, one immediately verifies that $T(\bar{\partial})$ does not depend on the choices of $s^{0}, s^{1} \ldots$ Therefore $T(\bar{\partial})$ is a non-zero element of $\operatorname{det}(E)$ canonically associated to $(E, \bar{\partial})$.

We shall call $T(\bar{\partial})$ the torsion of the acyclic complex $(E, \bar{\partial})$.

Let now $\left(E_{i}^{j}, \bar{\partial}, v\right)$ be a double complex, with $0 \leqq i \leqq m, 0 \leqq j \leqq l, v: E_{i}^{j} \rightarrow E_{i+1}^{j}$, and $\bar{\partial}: E_{i}^{j} \rightarrow E_{i}^{j+1}$. Let $\left(E_{i}, \bar{\partial}\right)$ (respectively $\left(E^{j}, v\right)$ ) denote the chain complex $\left(E_{i}^{j}\right)_{0 \leqq I \leqq I}$ respectively $\left(E_{i}^{j}\right)_{0 \leqq 1 \leqq m}$ ) with the chain map $\bar{\partial}$ (respectively $v$ ). Let $\lambda_{i}$ (respectively $\lambda^{j}$ ) be the determinant of $\left(E_{i}, \bar{\partial}\right)$ (respectively $\left(E^{j}, v\right)$ ). The complex lines

and

$$
\lambda_{0} \otimes\left(\lambda_{1}\right)^{-1} \otimes \lambda_{2} \otimes \cdots
$$

$$
\lambda^{0} \otimes\left(\lambda^{1}\right)^{-1} \otimes \lambda^{2} \otimes \cdots
$$

are canonically isomorphic. We call them the determinant of the double complex $E$, denoted $\operatorname{det}(E)$.

If all the lines and columns of $E$ are acyclic, we can define the torsion elements $T_{i}(\bar{\partial}) \in \lambda_{i}$ and $T^{j}(v) \in \lambda^{j}$ for $0 \leqq i \leqq m, 0 \leqq j \leqq l$.

Using [KM, Proposition 1], one checks that

$$
T_{0}(\bar{\partial}) \otimes\left[T_{1}(\bar{\partial})\right]^{-1} \otimes T_{2}(\bar{\partial}) \otimes \cdots=T^{0}(v) \otimes\left[T^{1}(v)\right]^{-1} \otimes T^{2}(v) \otimes \cdots
$$

Assume $m=2$ and the lines of $E$ are exact, i.e. we have a short exact sequence of chain complexes,

$$
0 \rightarrow E_{0} \underset{v}{\longrightarrow} E_{1} \longrightarrow \underset{v}{\longrightarrow} E_{2} \rightarrow 0 .
$$

Then $T^{0}(v) \otimes\left[T^{1}(v)\right]^{-1} \otimes T^{2}(v)$ is a canonical non-zero element in $\operatorname{det}(E)=$ $\lambda_{0} \otimes\left(\lambda_{1}\right)^{-1} \otimes \lambda_{2}$. It provides a canonical isomorphism

$$
\lambda_{1} \simeq \lambda_{0} \otimes \lambda_{2} \text {. }
$$

When $E_{2}$ is acyclic, $T_{2}(\bar{\partial})$ is non-zero in $\lambda_{2}$ and we get a canonical isomorphism,

$$
\lambda_{0} \simeq \lambda_{0} \otimes \lambda_{2}
$$

by sending $s \in \lambda_{0}$ to $s \otimes T_{2}(\bar{\partial})$. Therefore $\lambda_{1}$ is canonically isomorphic to $\lambda_{0}$.

When $E_{0}$ and $E_{2}$ are acyclic, the same is true for $E_{1}$ and the isomorphism $\lambda_{0} \simeq \lambda_{1}$ maps $T_{0}(\bar{\partial})$ to $T_{1}(\bar{\partial})$. 
Remark 1.2. A theory of determinants has been developed by Grothendieck and Knudsen-Mumford [KM]. We refer the reader to [KM] and to [BGS3] for more details.

Let us point out that in [KM], det $E$ is defined as the pair $\left(\Lambda^{n} E, n\right)$ of the line $\Lambda^{n} E$ with the integer $n \in Z$. The definition avoids the contradictions which might arise from sign problems. For instance, if $\operatorname{dim} E=n$ and $\operatorname{dim} F=m$, the isomorphism

$$
\operatorname{det}(E \oplus F) \simeq \operatorname{det}(F \oplus E),
$$

sending $e_{1} \wedge \cdots \wedge e_{n} \wedge f_{1} \wedge \cdots \wedge f_{m}$ to $f_{1} \wedge \cdots \wedge f_{m} \wedge e_{1} \wedge \cdots e_{n}$ is the multiplication by $(-1)^{n+m}$. Therefore the induced isomorphism

$$
\operatorname{det}(E) \otimes \operatorname{det}(F) \simeq \operatorname{det}(F) \otimes \operatorname{det}(E)
$$

makes sense for graded lines as in [KM, p 20], but not in our case.

However, most often these sign problems will not occur below and we shall neglect them. For us $\operatorname{det}(E)$ will just be $\Lambda^{n} E$.

2. Determinant and Cohomology of a Chain Complex. Let

$$
E: 0 \rightarrow E^{0} \underset{\bar{\partial}}{\longrightarrow} E^{1} \underset{\bar{\partial}}{\longrightarrow} \cdots \underset{\bar{\partial}}{\longrightarrow} E^{l} \rightarrow 0
$$

be an arbitrary chain complex of finite dimensional complex vector spaces. Let $\bar{\partial}_{i}=\bar{\partial}$ on $E^{i}$. Consider the cohomology of $E$ :

$$
H^{i}=\operatorname{Ker}\left(\bar{\partial}_{i}\right) / \operatorname{Im}\left(\bar{\partial}_{i-1}\right), \quad i=0, \ldots, l .
$$

For $0 \leqq i \leqq l$, there are exact sequences

$$
\begin{aligned}
& 0 \rightarrow \bar{\partial}\left(E^{i}\right) \rightarrow \operatorname{Ker}\left(\bar{\partial}_{i+1}\right) \rightarrow H^{i+1} \rightarrow 0, \\
& 0 \rightarrow \operatorname{Ker}\left(\bar{\partial}_{i+1}\right) \rightarrow E^{i+1} \underset{\bar{\partial}}{\longrightarrow} \bar{\partial}\left(E^{i+1}\right) \rightarrow 0 .
\end{aligned}
$$

The torsions of these exact sequences provide canonical isomorphisms

$$
\begin{aligned}
\operatorname{det}\left(E^{i+1}\right) & \simeq \operatorname{det}\left(\operatorname{Ker} \bar{\partial}_{i+1}\right) \otimes \operatorname{det}\left(\bar{\partial}\left(E^{i+1}\right)\right) \\
\operatorname{det}\left(\operatorname{Ker} \bar{\partial}_{i+1}\right) & \simeq \operatorname{det}\left(\bar{\partial}\left(E^{i}\right)\right) \otimes \operatorname{det}\left(H^{i+1}\right) .
\end{aligned}
$$

Therefore

$$
\operatorname{det}\left(E^{i+1}\right) \simeq \operatorname{det}\left(\bar{\partial}\left(E^{i}\right)\right) \otimes \operatorname{det}\left(H^{i+1}\right) \otimes \operatorname{det}\left(\bar{\partial}\left(E^{i+1}\right)\right) .
$$

We then obtain a canonical isomorphism

$$
\begin{gathered}
\left(\operatorname{det} E^{0}\right)^{-1} \otimes \operatorname{det} E^{1} \otimes\left(\operatorname{det} E^{2}\right)^{-1} \cdots \simeq\left(\operatorname{det} H^{0}\right)^{-1} \\
\otimes \operatorname{det} H^{1} \otimes\left(\operatorname{det} H^{2}\right)^{-1} \otimes \cdots .
\end{gathered}
$$

Let $A$ be a linear mapping acting on each $E^{i}, 0 \leqq i \leqq l$, and such that $A \bar{\partial}=\bar{\partial} A$. Then $A$ acts upon each cohomology group $H^{i}$. Let $\operatorname{Tr}_{E^{i}}(A)$ and $\operatorname{Tr}_{H^{i}}(A)$ be the trace of $A$ on $E^{i}$ and $H^{i}$ respectively.

Proposition 1.3. The following identity holds:

$$
\sum_{i=0}^{l}(-1)^{i} \operatorname{Tr}_{E^{i}}(A)=\sum_{i=0}^{l}(-1)^{i} \operatorname{Tr}_{H^{i}}(A)
$$


Proof. Using the exact sequences (1.4) we get

$$
\begin{aligned}
& \operatorname{Tr}_{\bar{\partial}\left(E^{i}\right)}(A)-\operatorname{Tr}_{\operatorname{Ker}\left(\bar{\partial}_{i+1}\right)}(A)+\operatorname{Tr}_{H^{i+1}}(A)=0, \\
& \operatorname{Tr}_{\operatorname{Ker}\left(\overline{\bar{o}}_{i+1}\right)}(A)-\operatorname{Tr}_{E^{i+1}}(A)+\operatorname{Tr}_{\overline{\hat{\alpha}}\left(E^{l+1}\right)}(A)=0 .
\end{aligned}
$$

Summing these equalities we get the Proposition.

3. Torsion and Analytic Torsion of an Hermitian Chain Complex. We still consider the chain complex (1.1).

Set

$$
\begin{aligned}
E^{+} & =\bigoplus_{i \text { even }} E^{i}, \quad E^{-}=\bigoplus_{i \text { odd }} E^{i}, \quad E=E^{+} \oplus E^{-}, \\
\lambda^{\prime} & =\left(\operatorname{det} E^{+}\right)^{-1} \otimes \operatorname{det} E^{-} .
\end{aligned}
$$

Let $i$ be the canonical isomorphisms from $\lambda$ into $\lambda^{\prime}$.

$s=s_{0}^{-1} \otimes s_{1} \otimes \cdots \in \lambda \rightarrow i(s)=\left(s_{0} \wedge s_{2} \cdots\right)^{-1} \otimes\left(s_{1} \wedge s_{3} \wedge \cdots\right) \in \lambda^{\prime}$.

Assume that the $E^{\imath}$ are Hermitian vector spaces. $E^{+}, E^{-}$are naturally endowed with Hermitian products so that the various $E^{i}$ are mutually orthogonal. $\lambda$ and $\lambda^{\prime}$ inherit the corresponding Hermitian metrics || and $\mid l^{\prime}$, and $i$ is an isometry from $\lambda$ into $\lambda^{\prime}$.

Let $\bar{\partial}^{*}$ be the adjoint of $\bar{\partial}$. Let $D \in \operatorname{End}(E)$ be given by:

$$
D=\bar{\partial}+\bar{\partial}^{*},
$$

and let $D_{ \pm}$be the restriction of $D$ to $E_{ \pm}$. If $s \in \operatorname{det} E_{+}, s \neq 0$, we $\operatorname{define} \operatorname{det} D_{+} \in \lambda^{\prime}$ by:

Clearly

$$
\operatorname{det} D_{+}=\left(s^{-1}\right) \otimes D_{+} s \text {. }
$$

$$
D^{2}=\left(\bar{\partial}+\bar{\partial}^{*}\right)^{2}=\bar{\partial} \bar{\partial}^{*}+\bar{\partial}^{*} \bar{\partial} .
$$

For $0 \leqq i \leqq n$, let $D_{i}^{2}$ be the restriction of $D^{2}$ to $E^{i}$. The norm of $\operatorname{det} D_{+}$in $\lambda^{\prime}$ is given by

$$
\left|\operatorname{det} D_{+}\right|=\left[\prod_{i \text { even }} \operatorname{det} D_{i}^{2}\right]^{1 / 2}=\left[\prod_{i \text { odd }} \operatorname{det} D_{i}^{2}\right]^{1 / 2} .
$$

We now assume that the chain complex (1.1) is acyclic.

Let us recall the definition of the analytic torsion of the chain complex (1.1) by Ray and Singer [RS].

Definition 1.4. The analytic torsion $\tau(\bar{\partial})$ of the acyclic chain complex (1.1) is the positive real number

$$
\tau(\bar{\partial})=\left\{\left(\operatorname{det} D_{1}^{2}\right)\left(\operatorname{det} D_{2}^{2}\right)^{-2}\left(\operatorname{det} D_{3}^{2}\right)^{3} \cdots\right\}^{1 / 2} .
$$

Proposition 1.5. The norm $|T(\bar{\partial})|$ of $T(\bar{\partial})$ in $\lambda$ is given by

Moreover

$$
|T(\bar{\partial})|=\tau(\bar{\partial}) \text {. }
$$

$$
i[T(\bar{\partial})]=\frac{|T(\bar{\partial})|}{\left|\operatorname{det} D_{+}\right|} \operatorname{det} D_{+}
$$

Proof. By splitting the acyclic Hermitian chain complex into the acyclic chain 
complexes corresponding to the eigenvalues $\lambda>0$ of $D^{2}$, we may and we will assume that $D^{2}$ has one single eigenvalue $\lambda>0$.

Each $E^{i}$ splits orthogonally into

$$
E^{i}=\bar{\partial}\left(E^{i-1}\right) \oplus \bar{\partial}^{*}\left(E^{i+1}\right)
$$

Take $s^{i} \neq 0$ in $\operatorname{det} \bar{\partial}^{*}\left(E^{i+1}\right)$. We define $s \in \operatorname{det} E^{+}$by

$$
s=s^{0} \wedge\left(\bar{\partial} s^{1} \wedge s^{2}\right) \wedge\left(\bar{\partial} s^{3} \wedge s^{4}\right) \wedge \cdots
$$

Then $s$ is non-zero. Moreover

$$
D_{+} s=\left(\bar{\partial} s^{0} \wedge \bar{\partial}^{*} \bar{\partial} s^{1}\right) \wedge\left(\bar{\partial} s^{2} \wedge \bar{\partial} * \bar{\partial} s^{3}\right) \wedge \cdots .
$$

Also $D^{2} s^{i}=\bar{\partial}^{*} \bar{\partial} s^{i}$. If $n_{i}=\operatorname{dim} \bar{\partial}^{*}\left(E^{i+1}\right)$, we find that

$$
D_{+} s=\lambda^{n_{1}+n_{3}+\cdot}\left(\bar{\partial} s^{0} \wedge s^{1}\right) \wedge\left(\bar{\partial} s^{2} \wedge s^{3}\right) \wedge \cdots,
$$

and so

$$
\begin{gathered}
s^{-1} \otimes D_{+} s=\lambda^{n_{1}+n_{3}+\cdots}\left(s^{0} \wedge\left(\bar{\partial} s^{1} \wedge s^{2}\right) \wedge \cdots\right)^{-1} \\
\otimes\left(\bar{\partial} s^{0} \wedge s^{1}\right) \wedge\left(\bar{\partial} s^{2} \wedge s^{3}\right) \cdots .
\end{gathered}
$$

We thus find that

$$
\operatorname{det} D_{+}=\lambda^{n_{1}+n_{3}+\cdots} i[T(\bar{\partial})] .
$$

Since $i$ is an isometry, (1.14) follows from (1.18).

Also the complex (1.1) being acyclic, $\operatorname{dim} E^{i}=n_{i}+n_{i-1}$.

Moreover

$$
\log \left|\operatorname{det} D_{+}\right|^{\prime 2}=\left(\operatorname{dim} E^{0}+\operatorname{dim} E^{2}+\cdots\right) \log \lambda .
$$

From (1.18), (1.19) we find

$\log |T(\bar{\partial})|^{2}=\left(n_{0}-n_{1}+n_{2} \cdots\right) \log \lambda=\left(\operatorname{dim} E^{1}-2 \operatorname{dim} E^{2}+3 \operatorname{dim} E^{3} \cdots\right) \log \lambda$,

from which we get (1.13).

\section{b) Number Operator and the Chern Character Forms of a Holomorphic}

\section{Hermitian Chain Complex}

Let $B$ denote a connected complex manifold of real dimension $n^{\prime}=2 l^{\prime}$, and let $J \in$ End $T B$ be the complex structure of $B$.

Let

$$
0 \rightarrow E_{0} \underset{v}{\longrightarrow} \rightarrow \cdots \underset{v}{\longrightarrow} E_{m} \rightarrow 0
$$

be a holomorphic chain complex of finite dimensional complex holomorphic vector bundles on $B$. In particular the chain map $v$ is holomorphic and $v^{2}=0$.

Set

$$
E_{+}=\bigoplus_{j \text { even }} E_{j}, \quad E_{-}=\bigoplus_{j \text { odd }} E_{j}, \quad E=E_{+} \oplus E_{-} .
$$


Let $N$ be the operator on $E$ which defines the $Z$ grading of $E$, i.e. $N$ is multiplication by $j$ on $E_{j}(0 \leqq j \leqq m)$. Similarly let $\tau$ be the operator defining the $Z_{2}$ grading of $E$, i.e. $\tau= \pm 1$ on $E_{ \pm}$.

End $E$ is a $Z_{2}$ graded algebra, the even (respectively odd) elements of End $E$ commuting (respectively anticommuting) with $\tau$.

The graded tensor product $\Lambda\left(T_{C}^{*} B\right) \hat{\otimes}$ End $E$ is also naturally $Z_{2}$ graded. If $A$, $A^{\prime} \in \Lambda\left(T_{C}^{*} B\right) \hat{\otimes}$ End $E$, we define the supercommutator $\left[A, A^{\prime}\right]$ by

$$
\left[A, A^{\prime}\right]=A A^{\prime}-(-1)^{\operatorname{deg} A \operatorname{deg} A^{\prime}} A^{\prime} A .
$$

In the sequel, $[A, B]$ will always denote the supercommutator of $A$ and $B$ (usually denoted $[A, B]_{s}$ ).

If $A \in$ End $E$, we define its supertrace $\operatorname{Tr}_{s} A$ by

$$
\operatorname{Tr}_{s} A=\operatorname{Tr}[\tau A] .
$$

As in Quillen [Q1], we extend $\operatorname{Tr}_{s}$ to $\Lambda\left(T_{C}^{*} B\right) \hat{\otimes}$ End $E$, with the convention that if $\eta \in \Lambda\left(T_{C}^{*} B\right)$ and $A \in$ End $E$,

$$
\operatorname{Tr}_{s}[\eta A]=\eta \operatorname{Tr}_{s} A \text {. }
$$

Recall that by [Q1] $\operatorname{Tr}_{s}$ vanishes on supercommutators.

We now assume that $E_{0}, \ldots, E_{m}$ and $E$ are endowed with smooth Hermitian products, and that $E$ is the orthogonal direct sum of the $E_{j}^{\prime}$ s.

Let $v^{*}$ be the adjoint of $v$. For $a \in C$, set

$$
V^{a}=a v+\bar{a} v^{*} .
$$

We will use the notation $V=V^{1}$.

Let $\nabla$ be the unique holomorphic Hermitian connection on $E$. The connection $\nabla$ splits into

$$
\nabla=\nabla^{\prime}+\nabla^{\prime \prime},
$$

where $\nabla^{\prime}, \nabla^{\prime \prime}$ are the holomorphic and antiholomorphic parts of $\nabla$.

As in [Q1], we will consider $\nabla, \nabla^{\prime}, \nabla^{\prime \prime}$ as being first order differential operators acting on smooth sections of $\Lambda\left(T_{C}^{*} B\right) \hat{\otimes} E$. In particular $\nabla^{2}$ is the curvature of $\nabla$. $V^{a}$ is odd in End $E . \nabla+V^{a}$ is a superconnection on $E$ in the sense of Quillen [Q1].

We first have the elementary result.

Proposition 1.6. The following relations hold:

$$
\begin{aligned}
{[\nabla, N] } & =0 \\
\left(\nabla^{\prime \prime}+a v\right)^{2} & =\left(\nabla^{\prime}+\bar{a} v^{*}\right)^{2}=0 . \\
\left(\nabla+V^{a}\right)^{2} & =\left[\nabla^{\prime}+\bar{a} v^{*}, \nabla^{\prime \prime}+a v\right], \\
{\left[\nabla^{\prime}+\bar{a} v^{*},\left(\nabla+V^{a}\right)^{2}\right] } & =\left[\nabla^{\prime \prime}+a v,\left(\nabla+V^{a}\right)^{2}\right]=0 . \\
{[v, N] } & =-v \\
{\left[v^{*}, N\right] } & =v^{*} .
\end{aligned}
$$

Proof. The operator $N$ is parallel with respect to $\nabla$ and so $[\nabla, N]=0$. Since $\nabla^{\prime \prime} v=0$, 
and $v^{2}=0$, we have

$$
\left(\nabla^{\prime \prime}+a v\right)^{2}=\nabla^{\prime \prime 2}+a^{2} v^{2}+a \nabla^{\prime \prime} v=0 .
$$

Similarly $\left(\nabla^{\prime}+\bar{a} v^{*}\right)^{2}=0$. The third and fourth line in (1.24) follow. The final two relations in (1.24) are obvious.

Remark 1.7. If $\lambda \in C$ and if $I$ is the identity mapping on $E$, we can replace $N$ by $N+\lambda I$ in Proposition 1.6, without changing the commutation relations.

By Quillen [Q1], we know that for any $a \in C, \operatorname{Tr}_{s}\left[\exp -\left(\nabla+V^{a}\right)^{2}\right]$ is a closed differential form, which represents in cohomology the (normalized) Chern character

$$
\overline{\operatorname{ch}}\left(E_{0}-E_{1}+E_{2} \cdots\right)=\overline{\operatorname{ch}} E_{0}-\overline{\operatorname{ch}} E_{1}+\overline{\operatorname{ch}} E_{2} \cdots
$$

(here $\overline{c h} E_{j}$ is the class in cohomology of $\operatorname{Tr}^{E_{j}}\left[\exp -\nabla^{2}\right]$ ).

Let

$$
\begin{aligned}
& T^{(1,0)} B=\left\{X \in T_{C} B ; \quad J X=i X\right\}, \\
& T^{(0,1)} B=\left\{X \in T_{C} B ; \quad J X=-i X\right\}
\end{aligned}
$$

be the holomorphic and antiholomorphic subbundles of the complex tangent bundle $T_{C}^{B}$. Let $T^{*(1,0)} B$ and $T^{*(0,1)} B$ be the corresponding dual spaces.

We denote by $\partial^{B}$ and $\bar{\partial}^{B}$ the usual derivation operators on the smooth sections of $\Lambda\left(T_{C}^{*} B\right)$.

Let $P$ be the subspace of smooth sections of $\Lambda\left(T_{C}^{*} B\right)$ made of sums of differentials of complex type $(p, p), p \geqq 0$. Let $P^{\prime} \subset P$ be the set of smooth forms $\omega$ in $P$ which can be written as $\omega=\partial^{B} \eta+\bar{\partial}^{B} \eta^{\prime}$ (where $\eta$ and $\eta^{\prime}$ are smooth differential forms).

When $\omega, \omega^{\prime} \in P$, we write $\omega \equiv \omega^{\prime}$ if $\omega-\omega^{\prime} \in P^{\prime}$. Note that if $\eta \in P$ is closed and has compact support and $\omega \equiv \omega^{\prime}$,

$$
\int_{B} \omega \wedge \eta=\int_{B} \omega^{\prime} \wedge \eta .
$$

So the pairing of elements of $P / P^{\prime}$ with such $\eta$ is well defined.

We first prove a simple result, which will be of constant use in the sequel.

Proposition 1.8. Let $A$ be the vector subspace of $\Lambda\left(T_{C}^{*} B\right) \hat{\otimes}$ End $E$ generated by smooth sections of $\Lambda^{(p, q)}\left(T_{C}^{*} B\right) \hat{\otimes} \operatorname{End}\left(E_{j}, E_{j+p-q}\right)$ for all $p, q, j \geqq 0$. Then $A$ is an algebra. Moreover if $\eta \in A, \operatorname{Tr}_{s} \eta \in P$.

Proof. It is clear that $A$ is an algebra. Since $\operatorname{Tr}_{s}$ vanishes on $\operatorname{End}\left(E_{j}, E_{k}\right)_{j \neq k}$, the end of the proposition is obvious.

We now prove a first double transgression formula.

Theorem 1.9. For any $a \in C$, the differential forms $\operatorname{Tr}_{s}\left[\exp -\left(\nabla+V^{a}\right)^{2}\right]$ and $\operatorname{Tr}_{s}\left[N \exp -\left(\nabla+V^{a}\right)^{2}\right]$ are in $P$ and only depend on $|a|$. Moreover $\operatorname{Tr}_{s}\left[\exp -\left(\nabla+V^{a}\right)^{2}\right]$ is closed. Also

$$
\begin{aligned}
& \frac{\partial}{\partial a} \operatorname{Tr}_{s}\left(\exp -\left(\nabla+V^{a}\right)^{2}\right)=-\partial^{B} \operatorname{Tr}_{s}\left(v \exp -\left(\nabla+V^{a}\right)^{2}\right), \\
& \frac{\partial}{\partial \bar{a}} \operatorname{Tr}_{s}\left(\exp -\left(\nabla+V^{a}\right)^{2}\right)=-\bar{\partial}^{B} \operatorname{Tr}_{s}\left(v^{*} \exp -\left(\nabla+V^{a}\right)^{2}\right),
\end{aligned}
$$




$$
\begin{aligned}
\operatorname{Tr}_{s}\left(a v \exp -\left(\nabla+V^{a}\right)^{2}\right) & =-\bar{\partial}^{B} \operatorname{Tr}_{s}\left(N \exp -\left(\nabla+V^{a}\right)^{2}\right) \\
\operatorname{Tr}_{s}\left(\bar{a} v^{*} \exp -\left(\nabla+V^{a}\right)^{2}\right) & =\partial^{B} \operatorname{Tr}_{s}\left(N \exp -\left(\nabla+V^{a}\right)^{2}\right)
\end{aligned}
$$

In particular

$$
\begin{aligned}
& \frac{\partial}{\partial a} \operatorname{Tr}_{s}\left(\exp -\left(\nabla+V^{a}\right)^{2}\right)=-\frac{1}{a} \bar{\partial}^{B} \partial^{B} \operatorname{Tr}_{s}\left(N \exp -\left(\nabla+V^{a}\right)^{2}\right), \\
& \frac{\partial}{\partial \bar{a}} \operatorname{Tr}_{s}\left(\exp -\left(\nabla+V^{a}\right)^{2}\right)=-\frac{1}{\bar{a}} \bar{\partial}^{B} \partial^{B} \operatorname{Tr}_{s}\left(N \exp -\left(\nabla+V^{a}\right)^{2}\right) .
\end{aligned}
$$

Proof. It is clear that if $\theta \in \mathbb{R}$,

$$
e^{i \theta N}\left(\nabla+V^{a}\right) e^{-i \theta N}=\nabla+V^{a e^{i \theta}}
$$

We conclude that $\operatorname{Tr}_{s}\left(\exp -\left(\nabla+V^{a}\right)^{2}\right)$ and $\operatorname{Tr}_{s}\left(N \exp -\left(\nabla+V^{a}\right)^{2}\right)$ are radial functions of $a$.

By (1.24) we have

$$
\left(\nabla+V^{a}\right)^{2}=\nabla^{2}+|a|^{2}\left(v v^{*}+v^{*} v\right)+a \nabla^{\prime} v+\bar{a} \nabla^{\prime \prime} v^{*} .
$$

So by Proposition 1.8, the considered differential forms are elements of $P$.

We know by [Q1] that $\operatorname{Tr}_{s}\left(\exp -\left(\nabla+V^{a}\right)^{2}\right)$ is closed. On $B \times C$ the form $\operatorname{Tr}_{s}\left[\exp -\left(\nabla+d a(\partial / \partial a)+d \bar{a}(\partial / \partial \bar{a})+V^{a}\right)^{2}\right]$ is also closed. It is equal to

$$
\operatorname{Tr}_{s} \exp \left(-\left(\nabla+V^{a}\right)^{2}-d a v-d \bar{a} v^{*}\right) .
$$
by

By Duhamel's formula, since $\operatorname{Tr}_{s}$ vanishes on supercommutators, (1.27) is given

$$
\begin{aligned}
& \operatorname{Tr}_{s}\left(\exp -\left(\nabla+V^{a}\right)^{2}\right)-d a \operatorname{Tr}_{s}\left(v \exp -\left(\nabla+V^{a}\right)^{2}\right) \\
& \quad-d \bar{a} \operatorname{Tr}_{s}\left(v^{*} \exp -\left(\nabla+V^{a}\right)^{2}\right)+d a d \bar{a} \varepsilon,
\end{aligned}
$$

where $\varepsilon$ is a differential form on $B$. Since (1.28) is closed under $\partial^{B}+d a(\partial / \partial a)$ and $\bar{\partial}^{B}+d \bar{a}(\partial / \partial \bar{a})$, we get the first two relations in $(1.25)$. On the other hand

$$
\begin{aligned}
d^{B} \operatorname{Tr}_{s}\left(N \exp -\left(\nabla+V^{a}\right)^{2}\right) & =\operatorname{Tr}_{s}\left[\nabla+V^{a}, N \exp -\left(\nabla+V^{a}\right)^{2}\right] \\
& =\operatorname{Tr}_{s}\left(\left[V^{a}, N\right] \exp -\left(\nabla+V^{a}\right)^{2}\right) \\
& =\operatorname{Tr}_{s}\left(\left(-a v+\bar{a} v^{*}\right) \exp -\left(\nabla+V^{a}\right)^{2}\right) .
\end{aligned}
$$

By a simple degree counting argument this implies the final formulas in (1.25). Finally (1.26) is an obvious consequence of (1.25).

We now prove a second series of results which are related to Theorem 1.9.

Theorem 1.10. For $a, b \in C$, the following identities hold:

$$
\begin{aligned}
& \partial^{B} \operatorname{Tr}_{s}\left(\exp \left(-\left(\nabla+V^{a}\right)^{2}+b N\right)\right)=b \bar{a} \operatorname{Tr}_{s}\left(v^{*} \exp \left(-\left(\nabla+V^{a}\right)^{2}+b N\right)\right) \\
& \bar{\partial}^{B} \operatorname{Tr}_{s}\left(\exp \left(-\left(\nabla+V^{a}\right)^{2}+b N\right)\right)=-b a \operatorname{Tr}_{s}\left(v \exp \left(-\left(\nabla+V^{a}\right)^{2}+b N\right)\right)
\end{aligned}
$$

Proof. Using (1.24), we find that

$$
\begin{aligned}
d^{B} \operatorname{Tr}_{s}\left(\exp \left(-\left(\nabla+V^{a}\right)^{2}+b N\right)\right) & =\operatorname{Tr}_{s}\left\{\left[\nabla+V^{a}, \exp \left(-\left(\nabla+V^{a}\right)^{2}+b N\right)\right]\right\} \\
& =b \operatorname{Tr}_{s}\left\{\left[V^{a}, N\right] \exp \left(-\left(\nabla+V^{a}\right)^{2}+b N\right)\right\} \\
& =b \operatorname{Tr}_{s}\left\{\left(-a v+\bar{a} v^{*}\right) \exp \left(-\left(\nabla+V^{a}\right)^{2}+b N\right)\right\} .
\end{aligned}
$$


Since $d^{B}=\partial^{B}+\bar{\partial}^{B}$, by an easy degree counting argument, we obtain (1.29).

We now prove that certain differential forms produced by the superconnection formalism are $\partial^{B}$ or $\bar{\partial}^{B}$ exact.

Let $\alpha_{1}, \alpha_{2}, \alpha_{3}$ denote the differential forms on $B$ which depend on $a \in C$ defined by the relation

$$
\begin{aligned}
& \operatorname{Tr}_{s}\left(N \exp -\left(\nabla+d a \frac{\partial}{\partial a}+d \bar{a} \frac{\partial}{\partial \bar{a}}+V^{a}\right)^{2}\right) \\
& \quad=\operatorname{Tr}_{s}\left(N \exp -\left(\nabla+V^{a}\right)^{2}\right)+d a \alpha_{1}+d \bar{a} \alpha_{2}+d a d \bar{a} \alpha_{3} .
\end{aligned}
$$

Since $\operatorname{Tr}_{s}\left(\exp \left(-\left(\nabla+V^{a}\right)^{2}+b N\right)\right)$ is a smooth function of $|a|^{2}$, there exist a smooth form $\beta_{1}(x, a, b)$ (for $\left.(x, a, b) \in B \times C^{2}\right)$ which depends smoothly on $|a|^{2}$ and is such that

$$
\operatorname{Tr}_{s}\left(\exp \left(-\left(\nabla+V^{a}\right)^{2}+b N\right)\right)=\operatorname{Tr}_{s}\left(\exp \left(-\nabla^{2}+b N\right)\right)+|a|^{2} \beta_{1} .
$$

Theorem 1.11. The following relation hold:

$$
\alpha_{1}=\bar{a} \bar{\partial}^{B}\left[\frac{1}{2} \frac{\partial^{2}}{\partial b^{2}} \beta_{1}\right]_{b=0}, \quad \alpha_{2}=-a \partial^{B}\left[\frac{1}{2} \frac{\partial^{2}}{\partial b^{2}} \beta_{1}\right]_{b=0} .
$$

Proof. Clearly

$$
\begin{aligned}
& \left.\frac{\partial}{\partial b} \operatorname{Tr}_{s}\left\{\exp \left(-\nabla+d a \frac{\partial}{\partial a}+d \bar{a} \frac{\partial}{\partial \bar{a}}+V^{a}\right)^{2}+b N\right)\right\}_{b=0} \\
& \quad=\operatorname{Tr}_{s}\left(N \exp -\left(\nabla+d a \frac{\partial}{\partial a}+d \bar{a} \frac{\partial}{\partial \bar{a}}+V^{a}\right)^{2}\right) .
\end{aligned}
$$

On the other hand if we expand

$$
\operatorname{Tr}_{s}\left\{\exp \left(-\left(\nabla+d a \frac{\partial}{\partial a}+d \bar{a} \frac{\partial}{\partial \bar{a}}+V^{a}\right)^{2}+b N\right)\right\}
$$

as in (1.28), the form which appears on the right of $d a$ is given by

$$
-\operatorname{Tr}_{s}\left(v \exp \left(-\left(\nabla+V^{a}\right)^{2}+b N\right)\right) \text {. }
$$

For $a \neq 0, b \neq 0$, by (1.29), (1.34) is equal to

$$
\frac{1}{b a} \bar{\partial}^{B} \operatorname{Tr}_{s}\left(\exp \left(-\left(\nabla+V^{a}\right)^{2}+b N\right)\right) \text {. }
$$

Also in (1.33), we can replace $\partial / \partial b$ by $\frac{1}{2}\left(\partial^{2} / \partial b^{2}\right) b$. We thus find that

$$
a \alpha_{1}=\bar{\partial}^{B} \frac{1}{2} \frac{\partial^{2}}{\partial b^{2}}\left(\operatorname{Tr}_{s}\left(\exp \left(-\left(\nabla+V^{a}\right)^{2}+b N\right)\right)_{b=0} .\right.
$$

By (1.36), we find that

$$
\bar{\partial}^{B} \frac{1}{2} \frac{\partial^{2}}{\partial b^{2}}\left(\operatorname{Tr}_{s}\left(\exp \left(-\nabla^{2}+b N\right)\right)\right)_{b=0}=0,
$$


and so

$$
a \alpha_{1}=\bar{\partial}^{B} \frac{1}{2} \frac{\partial^{2}}{\partial b^{2}} \operatorname{Tr}_{s}\left\{\exp \left(-\left(\nabla+V^{a}\right)^{2}+b N\right)-\exp \left(-\nabla^{2}+b N\right)\right\}_{b=0} .
$$

Using (1.31) and (1.38), we obtain (1.32). The second equation in (1.32) can be proved in the same way.

We now prove another basic identity. If $\eta \in \Lambda\left(T_{C}^{*} B\right) \hat{\otimes} C(d a, d \bar{a})$, we can expand $\eta$ in the form

$$
\eta=\eta_{0}+d a \eta_{1}+d \bar{a} \eta_{1}+d a d \bar{a} \eta_{3} ; \quad \eta_{i} \in \Lambda_{C}\left(T^{*} B\right), \quad 0 \leqq i \leqq 3 .
$$

Set

$$
[\eta]^{\text {dada }}=\eta_{3} .
$$

Theorem 1.12. For any $(a, b) \in C^{2}$,

$$
\begin{aligned}
& \frac{\partial}{\partial a} \operatorname{Tr}_{s}\left(\exp \left(-\left(\nabla+V^{a}\right)^{2}+b|a|^{2} N\right)\right)=-d^{B} \operatorname{Tr}_{s}\left(v \exp \left(-\left(\nabla+V^{a}\right)^{2}+b|a|^{2} N\right)\right) \\
& \quad+b \bar{a} \operatorname{Tr}_{s}\left\{\exp \left(-\left(\nabla+V^{a}\right)^{2}+\left(b|a|^{2}+d a d \bar{a}\right) N-a d a v-\bar{a} d \bar{a} v^{*}\right)\right\}^{d a d \bar{a}}
\end{aligned}
$$

In particular

$$
\begin{aligned}
& \frac{\partial}{\partial a}|a|^{2} \operatorname{Tr}_{s}\left(N \exp -\left(\nabla+V^{a}\right)^{2}\right)=-d_{B} \frac{\partial}{\partial b}\left[\operatorname{Tr}_{s}\left(v \exp \left(-\left(\nabla+V^{a}\right)^{2}+b|a|^{2} N\right)\right)\right]_{b=0} \\
& \quad+\bar{a} \operatorname{Tr}_{s}\left\{\exp \left(-\left(\nabla+V^{a}\right)^{2}+d a d \bar{a} N-a d a v-\bar{a} d \bar{a} v^{*}\right)\right\}^{\text {dada }} .
\end{aligned}
$$

Proof. Clearly, the left-hand side of (1.41) is the coefficient on the right of $d a$ in

$$
\left[d a \frac{\partial}{\partial a}, \operatorname{Tr}_{s}\left(\exp \left(-\left(\nabla+d a \frac{\partial}{\partial a}+V^{a}\right)^{2}+b|a|^{2} N\right)\right)\right] .
$$

Now (1.43) is given by

$$
\begin{aligned}
\operatorname{Tr}_{s}\{ & {\left[d a \frac{\partial}{\partial a},-\left(\nabla+d a \frac{\partial}{\partial a}+V^{a}\right)^{2}+b|a|^{2} N\right] } \\
& \left.\cdot \exp \left(-\left(\nabla+d a \frac{\partial}{\partial a}+V^{a}\right)^{2}+b|a|^{2} N\right)\right\} \\
= & \operatorname{Tr}_{s}\left\{\left(\left[\nabla+V^{a},\left(\nabla+d a \frac{\partial}{\partial a}+V^{a}\right)^{2}\right]+b \bar{a} d a N\right)\right. \\
& \left.\cdot \exp \left(-\left(\nabla+d a \frac{\partial}{\partial a}+V^{a}\right)^{2}+b|a|^{2} N\right)\right\} \\
= & \operatorname{Tr}_{s}\left\{\left(\left[\nabla+V^{a},\left(\nabla+d a \frac{\partial}{\partial a}+V^{a}\right)^{2}-b|a|^{2} N\right]\right.\right. \\
& \left.\left.+b|a|^{2}\left[V^{a}, N\right]+b \bar{a} d a N\right) \exp \left(-\left(\nabla+d a \frac{\partial}{\partial a}+V^{a}\right)^{2}+b|a|^{2} N\right)\right\}
\end{aligned}
$$




$$
\begin{aligned}
= & -d^{B} \operatorname{Tr}_{s}\left\{\exp \left(-\left(\nabla+d a \frac{\partial}{\partial a}+V^{a}\right)^{2}+b|a|^{2} N\right)\right\} \\
& +\operatorname{Tr}_{s}\left\{\left(b|a|^{2}\left(-a v+\bar{a} v^{*}\right)+b \bar{a} d a N\right)\right. \\
& \left.\cdot \exp \left(-\left(\nabla+d a \frac{\partial}{\partial a}+V^{a}\right)^{2}+b|a|^{2} N\right)\right\} .
\end{aligned}
$$

By selecting the factor of $d a$ in the left-hand side of (1.44), we find that

$$
\begin{aligned}
\frac{\partial}{\partial a} \operatorname{Tr}_{s}\left[\exp \left(-\left(\nabla+V^{a}\right)^{2}+b|a|^{2} N\right)\right]= & -d^{B} \operatorname{Tr}_{s}\left\{v \exp \left(-\left(\nabla+V^{a}\right)^{2}+b|a|^{2} N\right)\right\} \\
& +b \bar{a} \operatorname{Tr}_{s}\left\{\operatorname { e x p } \left(-\left(\nabla+V^{a}\right)^{2}+b|a|^{2} N\right.\right. \\
& -d a a v-d \bar{a}\left(-a v+\bar{a} v^{*}\right) \\
& +d a d \bar{a} N)\}^{d a d \bar{a}}
\end{aligned}
$$

Also

$$
d a a v+d \bar{a}\left(-a v+\bar{a} v^{*}\right)=(d a-d \bar{a}) a v+\bar{a} d \bar{a} v^{*}, \quad(d a-d \bar{a}) d \bar{a}=d a d \bar{a} .
$$

Then (1.41) follows from (1.45). By differentiating (1.41) at $b=0$, we obtain (1.42).

One verifies easily that

$$
\operatorname{Tr}_{s}\left(\exp \left(-\left(\nabla+V^{a}\right)^{2}+b|a|^{2} N\right)\right)
$$

is a smooth function of $|a|^{2}$. We can then write (1.46) in the form

$$
\operatorname{Tr}_{s}\left(\exp \left(-\left(\nabla+V^{a}\right)^{2}+b|a|^{2} N\right)\right)=\operatorname{Tr}_{s}\left(\exp \left(-\nabla^{2}\right)\right)+|a|^{2} \gamma_{0}(x, b)+|a|^{4} \gamma_{1}(x, a, b)
$$

where $\gamma_{0}$ (respectively $\gamma_{1}$ ) is a form on $B$ depending smoothly on $(x, b) \in B \times C$ (respectively on $\left.(x, a, b) \in B \times C^{2}\right)$.

Using Theorem 1.10, we now give a refined version of Theorem 1.12.

Theorem 1.13. For every $(a, b) \in C \times C^{*}$,

$$
\begin{aligned}
\frac{\partial}{\partial a} \operatorname{Tr}_{s} \exp \left(-\left(\nabla+V^{a}\right)^{2}+b|a|^{2} N\right)= & -\frac{\bar{a}}{b} \bar{\partial}^{B} \partial^{B} \gamma_{1}+b \bar{a}\left(\operatorname { T r } _ { s } \operatorname { e x p } \left(-\left(\nabla+V^{a}\right)^{2}\right.\right. \\
& \left.\left.+\left(b|a|^{2}+d a d \bar{a}\right) N-a d a v-\bar{a} d \bar{a} v^{*}\right)\right)^{\text {dad } \bar{a}}
\end{aligned}
$$

In particular

$$
\begin{aligned}
\frac{\partial}{\partial a}\left[|a|^{2} \operatorname{Tr}_{s}\left(N \exp \left(-\left(\nabla+V^{a}\right)^{2}\right)\right)\right]= & -\bar{a} \bar{\partial}^{B} \partial^{B} \frac{1}{2}\left[\frac{\partial^{2}}{\partial b^{2}} \gamma_{1}\right]_{b=0} \\
& +\bar{a} \operatorname{Tr}_{s}\left\{\operatorname { e x p } \left(-\left(\nabla+V^{a}\right)^{2}+d a d \bar{a} N\right.\right. \\
& \left.\left.-a d a v-\bar{a} d \bar{a} v^{*}\right)\right\}^{\text {dada }} .
\end{aligned}
$$


Proof. By (1.29), we know that

$$
\vec{\partial}^{B} \operatorname{Tr}_{s}\left(\exp \left(-\left(\nabla+V^{a}\right)^{2}+b|a|^{2} N\right)\right)=-b|a|^{2} a \operatorname{Tr}_{s}\left\{v \exp \left(-\left(\nabla+V^{a}\right)^{2}+b|a|^{2} N\right)\right\} .
$$

Using (1.50), we find that

$$
\bar{\partial}^{B} \operatorname{Tr}_{s}\left[\exp -\nabla^{2}\right]=0 ; \quad \bar{\partial}^{B} \gamma_{0}=0 .
$$

Using (1.41), (1.50), we find that for $a \neq 0$,

$$
\begin{aligned}
\frac{\partial}{\partial a} \operatorname{Tr}_{s}\left(\exp \left(-\left(\nabla+V^{a}\right)^{2}+b|a|^{2} N\right)\right) \\
=-\frac{1}{b a|a|^{2}} \bar{\partial}^{B} \partial^{B} \operatorname{Tr}_{s}\left(\exp \left(-\left(\nabla+V^{a}\right)^{2}+b|a|^{2} N\right)\right) \\
\quad+b \bar{a} \operatorname{Tr}_{s}\left\{\operatorname { e x p } \left(-\left(\nabla+V^{a}\right)^{2}\right.\right. \\
\left.\left.\quad+\left(b|a|^{2}+d a d \bar{a}\right) N-a d a v-\bar{a} d \bar{a} v^{*}\right)\right\}^{\text {dada }} .
\end{aligned}
$$

Using (1.51), we can replace the first term in the left-hand side of (1.52) by

$$
\begin{aligned}
& -\frac{\bar{\partial}^{B} \partial^{B}}{b a|a|^{2}}\left[\operatorname{Tr}_{s}\left(\exp \left(-\left(\nabla+V^{a}\right)^{2}+b|a|^{2} N\right)\right)\right. \\
& \left.-\operatorname{Tr}_{s}\left(\exp \left(-\nabla^{2}\right)\right)-|a|^{2} \gamma_{0}\right]=-\frac{\bar{a}}{b} \bar{\partial}^{B} \partial^{B} \gamma_{1} .
\end{aligned}
$$

Equation (1.48) follows from (1.52) and (1.53).

By differentiating both sides of (1.48) in $b$ at $b=0$ and replacing $\partial / \partial b$ by $\frac{1}{2}\left(\partial^{2} / \partial b^{2}\right) b$ in the right-hand side of (1.48), we obtain (1.49).

Remark 1.14. In particular, we find from (1.42) that

$$
\frac{\partial}{\partial u}\left[u \operatorname{Tr}_{s}\left(N \exp -(\nabla+\sqrt{u} V)^{2}\right)\right]_{u=0}=\operatorname{Tr}_{s}\left[N \exp \left(-\nabla^{2}\right)\right]
$$

which is not really a surprising result.

It turns out that when dealing with infinite dimensional chain complexes, the analogous identity will be highly non-trivial, because certain singular terms will disappear as $u \downarrow \downarrow 0$ in (1.54).

In particular the term $\{\quad\}^{\text {dada }}$ is of utmost importance in [BGS2] and [BGS3].

\section{c) Double Transgression of the Chern Character Forms}

Recall that $V=v+v^{*}$. For $u \geqq 0$, let $A_{u}$ be the superconnection

$$
A_{u}=\nabla+\sqrt{u} V \text {. }
$$

We first note the following application of Theorem 1.9.

Theorem 1.15. For any $u \geqq 0$, the differential forms $\operatorname{Tr}_{s}\left[\exp \left(-A_{u}^{2}\right)\right]$ and 
$\operatorname{Tr}_{s}\left[N \exp \left(-A_{u}^{2}\right)\right]$ are in $P$. The form $\operatorname{Tr}_{s}\left[\exp \left(-A_{u}^{2}\right)\right]$ is closed. Moreover for $u>0$,

$$
\begin{aligned}
\frac{\partial}{\partial u} \operatorname{Tr}_{s}\left(\exp \left(-A_{u}^{2}\right)\right) & =-\frac{1}{2 u}\left(\partial^{B}+\bar{\partial}^{B}\right) \operatorname{Tr}_{s}\left(\sqrt{u} V \exp \left(-A_{u}^{2}\right)\right), \\
\operatorname{Tr}_{s}\left[\sqrt{u} V \exp \left(-A_{u}^{2}\right)\right] & =\left(\partial^{B}-\bar{\partial}^{B}\right) \operatorname{Tr}_{s}\left(N \exp \left(-A_{u}^{2}\right)\right) .
\end{aligned}
$$

In particular

$$
\frac{\partial}{\partial u} \operatorname{Tr}_{s}\left[\exp \left(-A_{u}^{2}\right)\right]=-\frac{1}{u} \bar{\partial}^{B} \partial^{B} \operatorname{Tr}_{s}\left(N \exp \left(-A_{u}^{2}\right)\right) .
$$

Proof. Equations (1.55), (1.56) are obvious consequences of (1.25), (1.26).

We now assume that the chain complex $(E, v)$ is acyclic.

The operator $V^{2}$ is then self-adjoint and positive definite. By Duhamel's formula, as $u \uparrow+\infty, \operatorname{Tr}_{s}\left[\exp \left(-A_{u}^{2}\right)\right]$ and $\operatorname{Tr}_{s}\left[N \exp -A_{u}^{2}\right]$ decay exponentially uniformly on compact subsets of $B$.

We now will write an integrated version of (1.56). For later purposes, we will use a zêta function approach to the integration of (1.56).

Definition 1.16. For $s \in C, \operatorname{Re}(s)>0$, let $\zeta_{E}(s) \in P$ be defined by

$$
\zeta_{E}(s)=-\frac{1}{\Gamma(s)} \int_{0}^{+\infty} u^{s-1} \operatorname{Tr}_{s}\left[N \exp \left(-A_{u}^{2}\right)\right] d u .
$$

$\zeta_{E}(s)$ extends into a holomorphic function on $C$.

In particular

$$
\begin{aligned}
\zeta_{E}(0)= & -\operatorname{Tr}_{s}\left[N \exp -\nabla^{2}\right] \\
\zeta_{E}^{\prime}(0)= & -\int_{0}^{1}\left[\operatorname{Tr}_{s}\left(N \exp \left(-A_{u}^{2}\right)\right)-\operatorname{Tr}_{s}\left(N \exp \left(-\nabla^{2}\right)\right)\right] \frac{d u}{u} \\
& -\int_{1}^{+\infty} \operatorname{Tr}_{s}\left(N \exp \left(-A_{u}^{2}\right)\right) \frac{d u}{u}+\Gamma^{\prime}(1) \operatorname{Tr}_{s}\left(N \exp \left(-\nabla^{2}\right)\right) .
\end{aligned}
$$

Observe that the closed form $\operatorname{Tr}_{s}\left[N \exp \left(-\nabla^{2}\right)\right]$ is a generalized derived Euler characteristic of the chain complex $(E, v)$, since the component of degree 0 of $\operatorname{Tr}_{s}\left[N \exp \left(-\nabla^{2}\right)\right]$ is exactly the usual derived Euler characteristic

$$
-\operatorname{dim} E_{1}+2 \operatorname{dim} E_{2}-3 \operatorname{dim} E_{3} \ldots
$$

For $a \in C^{*}$, if $v$ is changed into $a v, V$ is changed into $V^{a}, \zeta_{E}(s)$ is changed into $|a|^{-2 s \zeta_{E}}(s)$ and $\zeta_{E}^{\prime}(0)$ is changed into

$$
\zeta_{E}^{\prime}(0)-2 \log |a| \zeta_{E}(0)=\zeta_{E}^{\prime}(0)+2 \log |a| \operatorname{Tr}_{s}\left[N \exp \left(-\nabla^{2}\right)\right] .
$$

Theorem 1.17. If the chain complex $(E, v)$ is acyclic, then

$$
\begin{aligned}
\int_{0}^{+\infty} \operatorname{Tr}_{s}\left(V \exp \left(-A_{u}^{2}\right)\right) \frac{d u}{\sqrt{u}} & =-\left(\partial^{B}-\bar{\partial}^{B}\right) \zeta_{E}^{\prime}(0), \\
\operatorname{Tr}_{s}\left[\exp \left(-\nabla^{2}\right)\right] & =-\bar{\partial}^{B} \partial^{B} \zeta_{E}^{\prime}(0) .
\end{aligned}
$$


Proof. Equation (1.60) is a trivial consequence of (1.55) and (1.56).

Remark 1.18. In Remark 1.7, we have seen that the commutation relations (1.24) still hold if $N$ is replaced by $N+\lambda I$ (with $\lambda \in C$ ). It is easy to verify that when $N$ is changed into $N+\lambda I, \zeta_{E}^{\prime}(0)$ remains constant in $P / P^{\prime}$.

Remark 1.19. The second line of $(1.60)$ has a natural interpretation in degree $(1,1)$. Namely $\operatorname{Tr}_{s}\left[-\nabla^{2}\right]$ is the curvature of the holomorphic Hermitian line bundle

$$
\lambda=\left(\operatorname{det} E_{0}\right)^{-1} \otimes \operatorname{det} E_{1} \otimes\left(\operatorname{det} E_{2}\right)^{-1} \cdots .
$$

By Definition 1.1, $\lambda$ has a canonical holomorphic non-zero section $T(v)$. By Proposition 1.5, we know that if $\tau(v)$ is the analytic torsion of the chain complex $(E, v)$, then

$$
|T(v)|=\tau(v) .
$$

If $\zeta_{E}^{(0)}(s)$ is the component of degree 0 in $\zeta_{E}(s)$, one verifies trivially that

$$
\zeta_{E}^{(0)}(s)=-\operatorname{Tr}_{s}\left\{N\left[V^{2}\right]^{-s}\right\} .
$$

Using (1.12), we get

$$
\log [\tau(v)]^{2}=-\zeta_{E}^{\prime(0)}(0) .
$$

So in degree $(1,1)$, we obtain from $(1.60)$ the relation

$$
\operatorname{Tr}_{s}\left[-\nabla^{2}\right]=\bar{\partial}^{B} \partial^{B} \log |T(v)|^{2} .
$$

Thus $-\zeta_{E}^{\prime}(0)$ is the natural generalization in $P$ of the logarithm of the analytic torsion.

\section{d) Multiplicativity Property of the Generalized Analytic Torsion}

Consider a double chain complex $E$ of finite dimensional holomorphic Hermitian vector bundles

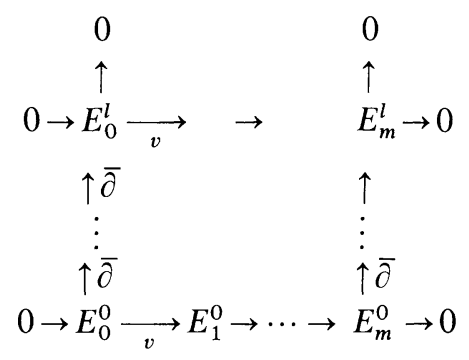

with holomorphic chain maps $\bar{\partial}$ and $v$ which are such that $\bar{\partial}^{2}=0, v^{2}=0, \bar{\partial} v+v \bar{\partial}=0$. The orthogonal direct sum $E=\oplus E_{i}^{j}$ is also a holomorphic Hermitian vector bundle on $B$.

Let $E_{i}$ (respectively $E^{j}$ ) be the chain complex of the $i^{\text {th }}$ column (respectively $j^{\text {th }}$ row) in (1.66) with the chain map $\bar{\partial}$ (respectively $v$ ).

The double chain complex $E$ carries naturally a horizontal grading, a vertical 
grading and a total grading. Let $N_{H}, N_{V}$ be the horizontal and vertical number operators. $N_{H}$ acts on $E_{i}$ by multiplication by $i$, and $N_{V}$ acts on $E^{j}$ by multiplication by $j$.

Then $N=N_{H}+N_{V}$ is the total number operator.

Let $\tau_{H}, \tau_{V}$ be the operators defining the horizontal and vertical $Z_{2}$ grading, i.e. $\tau_{H}= \pm 1$ on $E_{ \pm}^{j}, \tau_{V}= \pm 1$ on $E_{l}^{ \pm}$. Clearly

$$
\left[N_{H}, N_{V}\right]=0, \quad\left[\tau_{H}, \tau_{V}\right]=0 .
$$

Moreover $\tau=\tau_{H} \tau_{V}$ defines the total $Z_{2}$ grading of $E$, i.e. $\tau= \pm 1$ on $E_{ \pm}$.

The algebras $\Lambda\left(T_{C}^{*} B\right) \hat{\otimes}$ End $E_{i}, \Lambda\left(T_{C}^{*} B\right) \hat{\otimes}$ End $E^{j}, \quad \Lambda\left(T_{C}^{*} \bar{B}\right) \hat{\otimes}$ End $\bar{E} \quad$ are naturally endowed with supertraces, which we note $\operatorname{Tr}_{s}^{E_{t}}, \operatorname{Tr}_{s}^{E^{j}}$ and $\operatorname{Tr}_{s}$.

We still note $\nabla=\nabla^{\prime}+\nabla^{\prime \prime}$ the unique holomorphic Hermitian connection on every $E^{j}$.

If $\bar{\partial}^{*}, v^{*}$ are the adjoints of $\bar{\partial}, v$, the following commutation relations are verified

$$
[\bar{\partial}, v]=\left[\bar{\partial}^{*}, v^{*}\right]=0
$$

(remember that $[\bar{\partial}, v],\left[\bar{\partial}^{*}, v^{*}\right]$ are supercommutators).

Set

$$
D=\bar{\partial}+\bar{\partial}^{*}, \quad V=v+v^{*} .
$$

Assume that $\left(E_{i}, \bar{\partial}\right)(0 \leqq i \leqq m)$ and $\left(E^{j}, v\right)(0 \leqq j \leqq l)$ are everywhere acyclic.

Let $\tau_{i}(\bar{\partial})$ (respectively $\tau^{j}(v)$ ) be the analytic torsion of $\left(E_{i}, \bar{\partial}\right)$ (respectively $\left(E^{j}, v\right)$. It is a consequence of (1.3), (1.13) that

$$
\left[\tau_{0}(\bar{\partial})\right]\left[\tau_{1}(\bar{\partial})\right]^{-1}\left[\tau_{2}(\bar{\partial})\right] \cdots=\left[\tau^{0}(v)\right]\left[\tau^{1}(v)\right]^{-1}\left[\tau^{2}(v)\right] \cdots
$$

Using (1.64), we find that

$$
\Sigma(-1)^{i} \zeta_{E_{z}}^{(0)}(0)=\Sigma(-1)^{j \zeta_{E^{j}}^{(0)}}(0) .
$$

We will generalize (1.69) in any degree.

Theorem 1.20. Assume the rows and columns of $E$ are acyclic. Then

$$
\Sigma(-1)^{i} \zeta_{E_{l}}^{\prime}(0) \equiv \Sigma(-1)^{j} \zeta_{E^{j}}^{\prime}(0) .
$$

Proof. Let $\tilde{E}$ be the chain complex on $B \times C$ whose fiber at $(x, a) \in B \times C$ is $E_{x}$ with differential $\bar{\partial}+a v$. Since the columns of $E$ are acyclic, $\widetilde{E}$ is also acyclic.

Let $\zeta_{\tilde{E}}(s)$ be the zêta function associated to $\tilde{E}$, and $\phi_{a}: B \rightarrow B \times C$ the imbedding given by $\phi_{a}(x)=(x, a)$. When $\operatorname{Re}(s)>0$ we have

$$
\phi_{0}^{*}\left(\zeta_{\tilde{E}}(s)\right)=\sum_{i}(-1)^{i} \zeta_{E_{\imath}}(s)-\sum_{i} \frac{(-1)^{i}}{\Gamma(s)} i \int_{0}^{\infty} u^{s-1} \operatorname{Tr}_{s}^{E_{z}}\left(\exp -(\nabla+\sqrt{u} D)^{2}\right) d u
$$

Set

-for $u \geqq 1$,

$$
\varepsilon_{u}=\int_{u}^{+\infty} \frac{1}{h} \operatorname{Tr}_{s}^{E_{i}}\left[N_{V} \exp -(\nabla+\sqrt{h} D)^{2}\right] d h
$$


- for $u<1$

$$
\varepsilon_{u}=-\int_{0}^{u} \frac{1}{h} \operatorname{Tr}_{s}^{E_{i}}\left[N_{V}\left(\exp -(\nabla+\sqrt{h} D)^{2}-\exp \left(-\nabla^{2}\right)\right)\right] d h-\zeta_{E_{l}}^{\prime}(0) .
$$

As $u \uparrow \uparrow+\infty, \varepsilon_{u}$ decays exponentially.

Using (1.56) and (1.60), we find that for $\operatorname{Re}(s)>0$,

$$
\frac{1}{\Gamma(s)} \int_{0}^{+\infty} u^{s-1} \operatorname{Tr}_{s}^{E_{t}}\left(\exp -(\nabla+\sqrt{u} D)^{2}\right) d u=\bar{\partial}^{B} \partial^{B}\left[\frac{1}{\Gamma(s)} \int_{0}^{+\infty} u^{s-1} \varepsilon_{u} d u\right]
$$

Furthermore the differential form

$$
\eta(s)=\frac{1}{\Gamma(s)} \int_{0}^{+\infty} u^{s-1} \varepsilon_{u} d u
$$

extends holomorphically at $s=0$.

Using (1.71), (1.73), we find that

$$
\phi_{0}^{*}\left(\zeta_{\tilde{E}}^{\prime}(0)\right) \equiv \Sigma(-1)^{i} \zeta_{E_{\imath}}^{\prime}(0) .
$$

The proof will now consist in showing that for any $a \in C$,

$$
\phi_{a}^{*}\left(\zeta_{\tilde{E}}^{\prime}(0)\right) \equiv \phi_{0}^{*}\left(\zeta_{\tilde{E}}^{\prime}(0)\right)
$$

In particular (1.75) holds at $a=1$. By interchanging the roles of $\bar{\partial}$ and $v$, we will thus obtain (1.70).

So we now concentrate on the proof of (1.75).

By Theorem 1.17, we know that

$$
\bar{\partial}^{B \times C} \partial^{B \times C} \zeta_{\tilde{E}}^{\prime}(0)=-\operatorname{Tr}_{s}\left[\exp \left(-\nabla^{2}\right)\right] .
$$

In particular the right-hand side of (1.76) does not contain $d a$ or $d \bar{a}$.

Also there are differential forms on $B, \theta_{0}, \theta_{1}, \theta_{2}, \theta_{3}$ which depend smoothly on $(x, a) \in B \times C$, such that

$$
\zeta_{\tilde{E}}^{\prime}(0)=\theta_{0}+d a \theta_{1}+d \bar{a} \theta_{2}+d a d \bar{a} \theta_{3} .
$$

Clearly

$$
\theta_{0}=\phi_{a}^{*}\left[\zeta_{\tilde{E}}^{\prime}(0)\right]
$$

Since $\bar{\partial}^{B \times C} \partial^{B \times C} \zeta_{\tilde{E}}^{\prime}(0)$ does not contain any term involving dada , we find easily that

$$
\frac{\partial^{2} \theta_{0}}{\partial a \partial \bar{a}}-\partial^{B} \frac{\partial \theta_{1}}{\partial \bar{a}}-\bar{\partial}^{B} \frac{\partial \theta_{2}}{\partial a}-\bar{\partial}^{B} \partial^{B} \theta_{3}=0
$$

One readily verifies that $\theta_{0},\left(\partial \theta_{1} / \partial \bar{a}\right),\left(\partial \theta_{2} / \partial a\right), \theta_{3}$ are smooth functions of $|a|^{2}$ and thus smooth function of $|a|$. We will thus write $\theta_{0}(|a|),\left(\partial \theta_{1} / \partial \bar{a}\right)(|a|) \ldots$

Also if $f$ is a smooth function on $R^{+}$,

$$
\frac{\partial^{2}}{\partial a \partial \bar{a}} f(|a|)=\frac{1}{4}\left[f^{\prime \prime}(|a|)+\frac{1}{|a|} f^{\prime}(|a|)\right] .
$$


Using (1.79), (1.80) we find that there exists a form $H(x)$ on $B$ such that for $r>0$,

$$
\begin{aligned}
\frac{\partial \theta_{0}}{\partial r}(x, r)= & \frac{H(x)}{r}+\frac{4}{r} \partial^{B} \int_{0}^{r} \frac{\partial \theta_{1}}{\partial \bar{a}}(x, b) b d b \\
& +\frac{4}{r} \bar{\partial}^{B} \int_{0}^{r} \frac{\partial \theta_{2}}{\partial a}(x, b) b d b+\frac{4}{r} \bar{\partial}^{B} \partial^{B} \int_{0}^{r} \theta_{3}(x, b) b d b .
\end{aligned}
$$

Since $\left(\partial \theta_{0} / \partial r\right)(x, r)$ is smooth at $r=0$, we find that necessarily $H(x)=0$. It immediately follows from (1.81) that

$$
\theta_{0}(x, r) \equiv \theta_{0}(x, 0) \text {. }
$$

Using (1.82), we find that (1.75) has been proved. We have thus completed the proof of the theorem.

Remark 1.21. Take $a \in C^{*}$. If we change $v$ into $a v$, by (1.59), we know that $\zeta_{E^{j}}^{\prime}(0)$ is changed into $\zeta_{E^{j}}^{\prime}(0)+2 \log |a| \operatorname{Tr}_{s}^{E^{j}}\left[N_{H} \exp \left(-\nabla^{2}\right)\right]$.

On the other hand

$$
\Sigma(-1)^{j} \operatorname{Tr}_{s}^{E^{j}}\left[N_{H} \exp \left(-\nabla^{2}\right)\right]=\Sigma(-1)^{i} i \operatorname{Tr}_{s}^{E_{i}}\left[\exp \left(-\nabla^{2}\right)\right]
$$

Since the various $E_{i}$ are acyclic, we find that

$$
\Sigma(-1)^{j} \operatorname{Tr}_{s}^{E^{j}}\left[N_{H} \exp -\left(\nabla^{2}\right)\right] \equiv 0 .
$$

Equation (1.70) shows that in $P / P^{\prime} \Sigma(-1)^{j} \zeta_{E^{j}}^{\prime}(0)$ does not depend on $a \in C^{*}$, $a \neq 0$. Of course this fits with (1.83).

Let now

$$
\begin{aligned}
& E: 0 \rightarrow E_{0} \underset{v}{\longrightarrow} E_{1} \rightarrow \cdots \rightarrow E_{m} \rightarrow 0, \\
& E^{\prime}: 0 \rightarrow E_{m} \underset{v}{\longrightarrow} E_{m+1} \rightarrow \cdots \rightarrow E_{m+m^{\prime}} \rightarrow 0
\end{aligned}
$$

be two exact sequences of holomorphic Hermitian vector bundles with a holomorphic chain map $v$. In $E$ and $E^{\prime}, E_{m}$ is of course the same holomorphic Hermitian vector bundle.

Let $E^{\prime \prime}$ be the exact sequence of holomorphic Hermitian vector bundles,

$$
E^{\prime \prime}: 0 \rightarrow E_{0} \underset{v}{\longrightarrow} E_{1} \rightarrow \cdots \underset{v}{\longrightarrow} E_{m-1} \underset{v^{2}}{\longrightarrow} E_{m+1} \underset{v}{\longrightarrow} \cdots \underset{v}{\longrightarrow} E_{m+m^{\prime}} \rightarrow 0 .
$$

We now prove a property which generalizes a property of the standard analytic torsion.

Theorem 1.22. The following identity holds

$$
\zeta_{E^{\prime \prime}}^{\prime}(0) \equiv \zeta_{E}^{\prime}(0)+(-1)^{m+1} \zeta_{E^{\prime}}^{\prime}(0) .
$$

Proof. We first assume that $E^{\prime}$ is a short exact sequence, i.e. $m^{\prime}=2$. We thus consider the double complex 


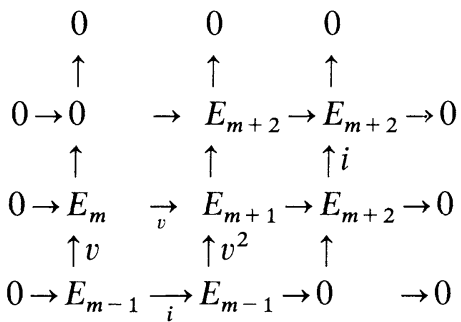

$$
\begin{aligned}
& \begin{array}{ccc}
\vdots & \vdots & \\
\uparrow v & \uparrow & \uparrow \\
0 \rightarrow E_{1} & \underset{i}{\rightarrow} E_{1} & \rightarrow 0 \rightarrow 0
\end{array} \\
& \begin{array}{ccc}
\uparrow v & \uparrow v & \uparrow \\
0 \rightarrow E_{0} & \rightarrow E_{0} & \rightarrow 0 \rightarrow 0
\end{array} \\
& \begin{array}{lll}
\uparrow & \uparrow & \uparrow \\
0 & 0 & 0
\end{array}
\end{aligned}
$$

The chain maps $E_{k} \rightarrow E_{k}$ are the identity maps $i$.

The rows and columns of (1.86) are trivially acyclic. We now use Theorem 1.19. Note that the rows and columns of the type $0 \rightarrow E_{k} \underset{i}{\longrightarrow} E_{k} \rightarrow 0$ have a zêta function which is exactly

$$
\frac{1}{\Gamma(s)} \int_{0}^{+\infty} u^{s-1} \operatorname{Tr}^{E_{k}}\left[\exp \left(-\nabla^{2}-u\right)\right] d u=\operatorname{Tr}^{E_{k}}\left[\exp -\left(\nabla^{2}\right)\right]
$$

So (1.87) is constant in $s$. Its derivative at 0 is 0 . So such lines or columns do not contribute to equality (1.70) for the double complex (1.86).

It is now easy to obtain (1.85) when $m^{\prime}=2$.

For general exact sequences $E^{\prime}$ we apply (1.85) repeatedly to short exact sequences and thus we obtain (1.85) in full generality.

\section{e) Bott-Chern Classes}

Let $E$ be a finite dimensional complex holomorphic vector bundle on the manifold $B$. Set $k=\operatorname{dim} E$.

Let $\mathbf{M}$ be the set of smooth Hermitian metrics on $E$. We endow $\mathbf{M}$ with the topology of uniform $C^{\infty}$ convergence on the compact subsets of $B$.

If $g \in \mathbf{M}, x \in B$, let $A_{x}^{g}$ be the subset of End $E_{x}$ of metrices which are Hermitian with respect to $g$.

If $g \in \mathbf{M}$, we can identify the tangent space $T_{g} \mathbf{M}$ with the vector space of smooth sections of $A^{g}$ on $B$. In fact a metric $g$ is an element of $\operatorname{End}\left(E, \bar{E}^{*}\right)$. If $h \in \operatorname{End}\left(E, \bar{E}^{*}\right)$ is an infinitesimal deformation of $g$ in $\mathbf{M}, g^{-1} h$ is the corresponding element in $A^{g}$.

Let $d^{\mathrm{M}}$ be the exterior differentiation operator on $\mathbf{M}$. We shall use below the one form $\eta=g^{-1} d^{\mathbf{M}} g$ on $\mathbf{M}$, with coefficients in $A$.

Let $X$ be the complex manifold of frames in $E$. $X$ is a principal $G L_{C}(k)$ bundle over $B$. Let $\pi$ be the projection $\mathbf{M} \times X \rightarrow B$. An element $u \in X$ is a linear isomorphism from $C^{k}$ into $E_{\pi u}$. 
Let $\theta$ be the equivariant representations of $\eta$. Namely $\theta$ is the 1 form on $\mathbf{M} \times X$ with values in $\operatorname{End}\left(C^{k}\right)$

$$
\theta=u^{-1} \pi^{*}\left(g^{-1} d^{\mathbf{M}} g\right) u
$$

If $g \in \mathbf{M}$, let $\omega^{g}$ be the unique holomorphic connection on $E$ which is Hermitian with respect to $g . \omega^{g}$ is a 1 form on $X$ with values in End $C^{k}$.

Let $\Omega^{g}$ be the corresponding curvature 2 form on $X$. We have the identities

$$
\partial^{X} \omega^{g}=-\left(\omega^{g}\right)^{2}, \quad \bar{\partial}^{X} \omega^{g}=\Omega^{g} .
$$

The space of connections is affine. So $d^{\mathbf{M}} \omega^{g}$ is a 2 form on $\mathbf{M} \times X$ taking its values in End $C^{k}$, and $d^{\mathbf{M}} \omega^{g}$ is the equivariant representation of a 2 form $\gamma$ on $\mathbf{M} \times B$ with values in End $E$.

Note that $\Lambda\left(T_{C}^{*} B\right) \hat{\otimes}$ End $E$ is a $Z_{2}$ graded algebra. We will use the notation [,] for supercommutators as in Sect. 1a).

We first have the elementary result.

Proposition 1.23. The following relations hold

$$
d^{\mathbf{M}} \theta=-\theta^{2}, \quad \partial^{X} \theta=-[\omega, \theta]-d^{\mathbf{M}} \omega .
$$

Proof. In local holomorphic coordinates, we know that $\omega^{g}=g^{-1} \partial^{B} g$. Also $\theta=g^{-1} d^{\mathbf{M}} g$.

Clearly

$$
\left(\partial^{X}+d^{\mathbf{M}}\right)\left(\theta+\omega^{g}\right)+\left(\theta+\omega^{g}\right)^{2}=0 .
$$

Equation (1.89) immediately follows.

Let $\nabla^{g}$ be the covariant differentiation operator corresponding to $\omega^{g}$. $\nabla^{g}$ splits into $\nabla^{g}=\nabla^{g^{\prime}}+\nabla^{g \prime \prime}$, where $\nabla^{g^{\prime}}$ is the holomorphic part of $\nabla^{g}$ and $\nabla^{g \prime \prime}$ the antiholomorphic part of $\nabla^{g}$.

$\Omega^{g}$ is the equivariant representation of the curvature tensor $\left(\nabla^{g}\right)^{2}$.

The scaled Chern character forms $\operatorname{Tr}\left[\exp -\left(\nabla^{g}\right)^{2}\right]$ can be pulled back to forms on $\mathbf{M} \times B$.

We now prove the basic result of Bott-Chern [Bot $C, 3.28$ ].

Theorem 1.24. The following identities hold

$$
\begin{aligned}
d^{\mathbf{M}} \operatorname{Tr}\left[\exp -\left(\nabla^{g}\right)^{2}\right] & =\bar{\partial}^{B} \operatorname{Tr}\left[\gamma \exp -\left(\nabla^{g}\right)^{2}\right], \\
\operatorname{Tr}\left[\gamma \exp -\left(\nabla^{g}\right)^{2}\right] & =-\partial^{B} \operatorname{Tr}\left[g^{-1} d^{\mathbf{M}} g \exp -\left(\nabla^{g}\right)^{2}\right] .
\end{aligned}
$$

In particular

$$
d^{\mathrm{M}} \operatorname{Tr}\left[\exp -\left(\nabla^{g}\right)^{2}\right]=-\bar{\partial}^{B} \partial^{B} \operatorname{Tr}\left[g^{-1} d^{\mathbf{M}} g \exp -\left(\nabla^{g}\right)^{2}\right] .
$$

Proof. For every $g \in \mathbf{M}, \omega^{g}$ is a one form on $X$. The pull back of $\omega^{g}$ to $\mathbf{M} \times X$ defines a connection $\omega$ on the bundle $E$ over $\mathbf{M} \times B$.

Using (1.88), we find that

$$
\left(d^{\mathbf{M}}+d^{X}\right) \omega=-\omega^{2}+d^{\mathbf{N i}} \omega+\Omega .
$$

Then $d^{\mathbf{M}} \omega+\Omega$ is the curvature form of $E$ on $\mathbf{M} \times X$. Therefore $\operatorname{Tr}\left[\exp -\left(\Omega+d^{\mathrm{M}} \omega\right)\right]$ is a closed form on $\mathbf{M} \times B$. 
Also by Duhamel's formula

$$
\operatorname{Tr}\left[\exp -\left(\Omega+d^{\mathbf{M}} \omega\right)\right]=\operatorname{Tr}[\exp -\Omega]-\operatorname{Tr}\left[d^{\mathbf{M}} \omega \exp (-\Omega)\right]+C,
$$

where $C$ is of degree $\geqq 2$ in the Grassmann variables on $\mathbf{M}$.

Since (1.92) is closed, the first line of (1.90) follows by a simple degree counting argument.

Let $z$ be an odd Grassmann variable.

Clearly

$$
\operatorname{Tr}[\exp (-\Omega+z \theta)]=\operatorname{Tr}[\exp -\Omega]+z \operatorname{Tr}[\theta \exp -\Omega] .
$$

On the other hand

$$
\partial^{X} \operatorname{Tr}[\exp (-\Omega+z \theta)]=\operatorname{Tr}\left[\left(-\partial^{X} \Omega-z \partial^{X} \theta\right) \exp (-\Omega+z \theta)\right] .
$$

By (1.88), we have

$$
\partial^{X} \Omega=[\Omega, \omega] .
$$

Using (1.89), (1.94) we find that

$\partial^{X} \operatorname{Tr}[\exp (-\Omega+z \theta)]=-\operatorname{Tr}([\omega,-\Omega+z \theta] \exp (-\Omega+z \theta))+z \operatorname{Tr}\left(d^{\mathrm{M}} \omega \exp (-\Omega)\right)$

The first term in the right-hand side of (1.96) clearly vanishes. Using (1.93), (1.96) and identifying the coefficient of $z$ we obtain the second line of (1.90). Equation (1.91) follows from (1.90).

The form $d^{\mathbf{M}} \operatorname{Tr}\left[\exp -\left(\nabla^{g}\right)^{2}\right]$ is the "gradient" of $\operatorname{Tr}\left[\exp -\left(\nabla^{g}\right)^{2}\right]$ on $\mathbf{M}$.

The question arises to know if $\operatorname{Tr}\left[g^{-1} d^{\mathbf{M}} g \exp -\left(\nabla^{g}\right)^{2}\right)$ is also a gradient on M. This question was first settled by Donaldson [D1, Proposition 6]. We here give a new proof of Donaldson's results.

$z$ again denotes an odd Grassmann variable.

We first prove an intermediary result.

Theorem 1.25. The following identity holds:

$$
\begin{aligned}
& d^{\mathrm{M}} \operatorname{Tr}\left[\exp \left(-\left(\nabla^{g}\right)^{2}+z g^{-1} d^{\mathbf{M}} g\right)\right]=\frac{1}{2} \partial^{B} \operatorname{Tr}\left[\left(\nabla^{g \prime \prime} g^{-1} d^{\mathbf{M}} g\right)\right. \\
& \left.\quad \cdot \exp \left(-\left(\nabla^{g}\right)^{2}+z g^{-1} d^{\mathbf{M}} g\right)\right]-\frac{1}{2} \bar{\partial}^{B} \operatorname{Tr}\left[\left(\nabla^{g^{\prime}} g^{-1} d^{\mathbf{M}} g\right)\right. \\
& \left.\quad \cdot \exp \left(-\left(\nabla^{g}\right)^{2}+z g^{-1} d^{\mathbf{M}} g\right)\right] .
\end{aligned}
$$

Proof. Clearly

$$
d^{\mathbf{M}} \operatorname{Tr}[\exp (-\Omega+z \theta)]=\operatorname{Tr}\left[\left(-d^{\mathbf{M}} \Omega-z d^{\mathbf{M}} \theta\right) \exp (-\Omega+z \theta)\right] .
$$

Using (1.88), (1.89), we find that

$$
d^{\mathrm{M}} \Omega=\bar{\partial}^{X} \partial^{X} \theta+[\Omega, \theta]-\left[\omega, \bar{\partial}^{x} \theta\right] .
$$

So from (1.89), (1.98), (1.99), we get

$$
\begin{aligned}
d^{\mathrm{M}} \operatorname{Tr}[\exp (-\Omega+z \theta)]= & \operatorname{Tr}\left[\left(-\bar{\partial}^{x} \partial^{X} \theta+\left[\omega, \bar{\partial}^{x} \theta\right]\right) \exp (-\Omega+z \theta)\right] \\
& +z \operatorname{Tr}\left[\theta^{2} \exp (-\Omega)\right]+\operatorname{Tr}([\theta, \Omega] \exp (-\Omega+z \theta)) .
\end{aligned}
$$


Since

$$
\operatorname{Tr}([\theta, \Omega-z \theta] \exp (-\Omega+z \theta))=0
$$

we find that

$$
\begin{aligned}
d^{\mathbf{M}} \operatorname{Tr}[\exp (-\Omega+z \theta)]= & \operatorname{Tr}\left[\left(-\bar{\partial}^{X} \partial^{X} \theta+\left[\omega, \bar{\partial}^{X} \theta\right]\right) \exp (-\Omega+z \theta)\right] \\
& -z \operatorname{Tr}\left[\theta^{2} \exp (-\Omega)\right] .
\end{aligned}
$$

Let $z^{\prime}$ be an even Grassmann variable (which is such that $z^{\prime 2}=0$, and which commutes with $\bar{\partial}^{B}, \partial^{B} \ldots$ and with $z$ ). Clearly

$$
\begin{aligned}
\operatorname{Tr}\left[\exp \left(-\Omega+z \theta+z^{\prime}\left(\partial^{X} \theta+[\omega, \theta]\right)\right)\right]= & \operatorname{Tr}[\exp (-\Omega+z \theta)] \\
& +z^{\prime} \operatorname{Tr}\left[\left(\partial^{X} \theta+[\omega, \theta]\right) \exp (-\Omega+z \theta)\right], \\
\operatorname{Tr}\left[\exp \left(-\Omega+z \theta+z^{\prime} \bar{\partial}^{X} \theta\right)\right]= & \operatorname{Tr}[\exp (-\Omega+z \theta)] \\
& +z^{\prime} \operatorname{Tr}\left[\bar{\partial}^{X} \theta \exp (-\Omega+z \theta)\right] .
\end{aligned}
$$

Using (1.88), (1.89) we find that

$$
\begin{aligned}
\bar{\partial}^{X} \operatorname{Tr} & {\left[\exp \left(-\Omega+z \theta+z^{\prime}\left(\partial^{X} \theta+[\omega, \theta]\right)\right)\right] } \\
= & \operatorname{Tr}\left[\left(-z \bar{\partial}^{X} \theta+z^{\prime}\left(\bar{\partial}^{X} \partial^{X} \theta+[\Omega, \theta]-\left[\omega, \bar{\partial}^{X} \theta\right]\right)\right)\right. \\
& \cdot \exp \left(-\Omega+z \theta+z^{\prime}\left(\partial^{X} \theta+[\omega, \theta]\right)\right] \\
= & z^{\prime} \operatorname{Tr}\left[\left(\bar{\partial}^{X} \partial^{X} \theta-\left[\omega, \bar{\partial}^{X} \theta\right]\right) \exp (-\Omega+z \theta)\right] \\
& +z^{\prime} \operatorname{Tr}[[\Omega, \theta] \exp (-\Omega+z \theta)] \\
& -z \operatorname{Tr}\left[\bar{\partial}^{X} \theta \exp \left(-\Omega+z^{\prime}\left(\partial^{X} \theta+[\omega, \theta]\right)\right)\right] .
\end{aligned}
$$

Using (1.100) again, we find that

$$
\begin{aligned}
& \bar{\partial}^{X} \operatorname{Tr}\left[\exp \left(-\Omega+z \theta+z^{\prime}\left(\partial^{X} \theta+[\omega, \theta]\right)\right]=z^{\prime} \operatorname{Tr}\left[\left(\bar{\partial}^{X} \partial^{X} \theta-\left[\omega, \bar{\partial}^{X} \theta\right]\right) \exp (-\Omega+z \theta)\right]\right. \\
& \quad+2 z^{\prime} z \operatorname{Tr}\left[\theta^{2} \exp -\Omega\right]-z \operatorname{Tr}\left[\bar{\partial}^{X} \theta \exp \left(-\Omega+z^{\prime}\left(\partial^{X} \theta+[\omega, \theta]\right)\right)\right] .
\end{aligned}
$$

Similarly, by (1.88), (1.89), we get

$$
\begin{aligned}
& \partial^{X} \operatorname{Tr} \exp \left(-\Omega+z \theta+z^{\prime} \bar{\partial}^{X} \theta\right)=\operatorname{Tr}\left[\partial^{X}+\omega, \exp \left(-\Omega+z \theta+z^{\prime} \bar{\partial}^{X} \theta\right)\right] \\
& \quad=\operatorname{Tr}\left[\left(z^{\prime} \partial^{X} \bar{\partial}^{X} \theta+z^{\prime}\left[\omega, \bar{\partial}^{X} \theta\right]-z\left(\partial^{X} \theta+[\omega, \theta]\right)\right) \exp \left(-\Omega+z \theta+z^{\prime} \bar{\partial}^{X} \theta\right)\right],
\end{aligned}
$$

or equivalently

$$
\begin{aligned}
\partial^{X} \operatorname{Tr}\left[\exp \left(-\Omega+z \theta+z^{\prime} \bar{\partial}^{X} \theta\right)\right]= & z^{\prime} \operatorname{Tr}\left[\left(-\bar{\partial}^{X} \partial^{X} \theta+\left[\omega, \bar{\partial}^{X} \theta\right]\right) \exp (-\Omega+z \theta)\right] \\
& -z \operatorname{Tr}\left[\left(\partial^{X} \theta+[\omega, \theta]\right) \exp \left(-\Omega+z^{\prime} \bar{\partial}^{X} \theta\right)\right] .
\end{aligned}
$$

If $A=B+C z^{\prime}$, set $C=A^{z^{\prime}}$. One immediately verifies that

$$
\operatorname{Tr}\left[\bar{\partial}^{X} \theta \exp \left(-\Omega+z^{\prime}\left(\partial^{X} \theta+[\omega, \theta]\right)\right)\right]^{z^{\prime}}=\operatorname{Tr}\left[\left(\partial^{X} \theta+[\omega, \theta]\right) \exp \left(-\Omega+z^{\prime} \bar{\partial}^{X} \theta\right)\right]^{z^{\prime}} .
$$

Using (1.103), (1.105), (1.106) and identifying the terms containing $z^{\prime}$, we get

$$
\begin{aligned}
& \frac{1}{2} \partial^{X} \operatorname{Tr}\left[\bar{\partial}^{X} \theta \exp (-\Omega+z \theta)\right]-\frac{1}{2} \bar{\partial}^{X} \operatorname{Tr}\left[\left(\partial^{X} \theta+[\omega, \theta]\right) \exp (-\Omega+z \theta)\right] \\
& \quad=\operatorname{Tr}\left[\left(-\bar{\partial}^{X} \partial^{X} \theta+\left[\omega, \bar{\partial}^{X} \theta\right]\right) \exp (-\Omega+z \theta)\right]-z \operatorname{Tr}\left[\theta^{2} \exp -\Omega\right] .
\end{aligned}
$$


Comparing with (1.101), we obtain (1.97).

We now consider

$$
\operatorname{Tr}\left[g^{-1} d^{\mathbf{M}} g \exp -\left(\nabla^{g}\right)^{2}\right]
$$

as a one form on $\mathbf{M}$ with values in $P$.

In particular, we find from (1.97) that

or equivalently that

$$
d^{\mathrm{M}} \operatorname{Tr}\left[g^{-1} d^{\mathrm{M}} g \exp -\left(\nabla^{g}\right)^{2}\right] \in P^{\prime},
$$

$$
d^{\mathbf{M}} \operatorname{Tr}\left[g^{-1} d^{\mathbf{M}} g \exp -\left(\nabla^{g}\right)^{2}\right] \equiv 0 .
$$

Therefore $\operatorname{Tr}\left[g^{-1} d^{\mathbf{M}} g \exp -\left(\nabla^{g}\right)^{2}\right]$ is a closed one form on $\mathbf{M}$ with values in $P / P^{\prime}$.

Since $\mathbf{M}$ is convex, a closed one form on $\mathbf{M}$ is exact. We will then integrate this form.

We fix a metric $g_{0}$ in $\mathbf{M}$. Take $g \in \mathbf{M}$, and let $g_{t}(0 \leqq t \leqq 1)$ be any smooth path in $\mathbf{M}$ connecting $g_{0}$ and $g$.

Definition 1.26. We define $f(g)$ in $P / P^{\prime}$ by

$$
f(g)=-\int_{0}^{1} \operatorname{Tr}\left[g_{t}^{-1} d^{\mathbf{M}} g_{t} \exp -\left(\nabla^{g_{t}}\right)^{2}\right] d t .
$$

Clearly, $f(g)$ does not depend on the path connecting $g_{0}$ and $g$ and moreover

$$
d^{\mathbf{M}} f \equiv-\operatorname{Tr}\left[g^{-1} d^{\mathbf{M}} g \exp -\left(\nabla^{g}\right)^{2}\right] .
$$

Note that the operator $\bar{\partial}^{B} \partial^{B}$ acts naturally on $P / P^{\prime}$.

Theorem 1.27. For any $g \in \mathbf{M}$

$$
\operatorname{Tr}\left[\exp -\left(\nabla^{g}\right)^{2}\right]-\operatorname{Tr}\left[\exp -\left(\nabla^{g 0}\right)^{2}\right]=\bar{\partial}^{B} \partial^{B} f(g) .
$$

Proof. This is obvious by (1.91) and (1.112).

Remark 1.28. The definition of $f(g)$ and Theorem 1.27 were given by Bott and Chern [Bot C, 3.28]. The fact that $f(g)$ does not depend (modulo $P^{\prime}$ ) on the path joining $g_{0}$ to $g$ was first noticed by Donaldson, [D1, Prop. 6].

As in [Bot C] and [D1], we remark that the definition of $f(g)$ and the whole Sect. 1e) are valid for any characteristic class and not only for the Chern character. More precisely, let $\phi: M_{k}(\mathbf{C}) \rightarrow \mathbf{C}$ be any polynomial map on $(k, k)$ matrices (or more generally a formal power series) invariant under conjugation by $G L_{k}(\mathbf{C})$. Then (1.91) can be generalized to

$$
d^{\mathrm{M}} \phi(\Omega)=\bar{\partial}^{B} \partial^{B}\left\langle\phi^{\prime}(\Omega), \theta\right\rangle .
$$

Furthermore, (1.97) can be extended to a general $\phi$. In particular (1.106) merely expresses the fact that two cross-derivations are equal. Definition 1.26 immediately extends to any such $\phi$, and (1.113) is now

$$
\phi\left(-\left(\nabla^{g}\right)^{2}\right)-\phi\left(-\left(\nabla^{g_{0}}\right)^{2}\right)=\bar{\partial}^{B} \partial^{B} f(g) .
$$

In [BGS2], this will be applied to the case where $\phi$ is the Todd genus $T d$. 


\section{f) Axiomatic Definition of the Secondary Classes of Bott and Chern}

Let $\phi: M_{k}(\mathbf{C}) \rightarrow \mathbf{C}$ be, as in Remark 1.28, an invariant polynomial map and

$$
E: 0 \rightarrow E_{0} \underset{v}{\longrightarrow} E_{1} \underset{v}{\longrightarrow} \cdots \underset{v}{\longrightarrow} E_{m} \rightarrow 0
$$

an acyclic complex of holomorphic vector bundles on a complex manifold $B$, equipped with Hermitian metrics $g_{j}, j=0, \ldots, m$. We assume that every $E_{j}$ has rank less than or equal to $k$. Let $\Omega_{j}$ be the curvature form of $\left(E_{j}, g_{j}\right)$ and $\phi\left(E_{j}\right) \in P$ the form $\phi\left(-\Omega_{j} / 2 \pi i\right)$.

Let $F_{0}=0$ and $F_{j}=v\left(E_{j-1}\right)$ if $j>0$. We endow $F_{j}$ with the metric $g_{j}$. From $E$, we get short exact sequences

$$
S_{j}: 0 \rightarrow F_{j} \rightarrow E_{j} \rightarrow F_{j+1} \rightarrow 0 .
$$

We say that $E$ is split if, for every $j \geqq 0$, the exact sequence $S_{j}$ admits an holomorphic splitting which makes $E_{j}$ isometric to the orthogonal direct sum $F_{j} \oplus F_{j+1}$.

Theorem 1.29. Fix an integer $m>0$. There exists a unique way to attach to every exact sequence $E$ as above a class $\tilde{\phi}(E)$ in $P / P^{\prime}$ such that:

i) $(1 / 2 \pi i) \partial^{B} \bar{\partial}^{B} \tilde{\phi}(E)=\sum_{j=0}^{m}(-1)^{j}\left(\phi\left(E_{j}\right)-\phi\left(F_{j} \oplus F_{j+1}\right)\right)$.

ii) For every map of complex manifolds $f: B^{\prime} \rightarrow B$,

$$
\tilde{\phi}\left(f^{*}(E)\right)=\left(f^{*}(\tilde{\phi}(E)) .\right.
$$

iii) If $E$ is split, $\tilde{\phi}(E)=0$.

Proof. Let $\mathbf{P}^{1}$ be the complex projective line. Given $E$ as above, we shall define an acyclic complex $\widetilde{E}$ on $B \times \mathbf{P}^{1}$. Let $\mathcal{O}(1)$ be the standard line bundle of degree one on $\mathbf{P}^{1}$ and $\sigma$ a section of $\mathcal{O}(1)$ which vanishes only at $\infty$. Given $n \geqq 0$ and a bundle $F$ on $B \times \mathbf{P}^{1}$ we define $F(n)$ by the formulae

$$
F(0)=F \quad \text { and } \quad F(n)=F(n-1) \otimes \mathcal{O}(1) .
$$

When $j=0, \ldots, m-1$ the bundle $F_{j}$ can be mapped diagonally into $E_{j} \oplus F_{i}(1)$ by the inclusion into $E_{j}$ and by $\mathbf{i d}_{F_{j}} \otimes \sigma$ into $F_{j}(1)$.

Define

$$
\tilde{E}_{j}=\operatorname{Coker}\left(F_{j} \rightarrow E_{j} \oplus F_{j}(1)\right)(m-j),
$$

and

$$
\tilde{F}_{j}=F_{j}(m+1-j) .
$$

The bundle $\widetilde{E}_{j}$ maps onto $\left(E_{j} / F_{j}\right)(m-j)=\widetilde{F}_{j+1}$, and we get exact sequences

$$
\tilde{S}_{j}: 0 \rightarrow \tilde{F}_{j} \rightarrow \tilde{E}_{j} \rightarrow \tilde{F}_{j+1} \rightarrow 0 .
$$

These patch together to give a long exact sequence

$$
\tilde{E}: 0 \rightarrow \widetilde{E}_{0} \rightarrow \widetilde{E}_{1} \rightarrow \cdots \rightarrow \widetilde{E}_{m} \rightarrow 0 .
$$

For every point $z \in \mathbf{P}^{1}$, let $i_{z}: B \rightarrow B \times \mathbf{P}^{1}$ be the map sending $y \in B$ to $(y, z) \in B \times \mathbf{P}^{1}$. When $z \neq \infty$, we have $\sigma(z) \neq 0$, hence $i_{z}^{*}(\tilde{E})$ is isomorphic to $E$. On the other hand $i_{\infty}^{*}\left(\widetilde{E}_{j}\right) \simeq F_{j} \oplus F_{j+1}$. Using a partition of unity we can choose a metric $\tilde{g}_{j}$ on $\widetilde{E}_{j}$ in 
such a way that the isomorphisms $i_{0}^{*}\left(\tilde{E}_{j}\right) \simeq E_{j}$ and $i_{\infty}^{*}\left(\bar{E}_{j}\right) \simeq F_{j} \oplus F_{j+1}$ become isometries. Therefore, as acyclic complexes of Hermitian holomorphic vector bundles, $i_{0}^{*}(\tilde{E})$ is equal to $E$ and $i_{\infty}^{*}(\tilde{E})$ is split.

To define a class $\tilde{\phi}(\tilde{E})$ having properties i), ii) and iii), we consider the integrable function $\log |z|^{2}$ on $\mathbf{P}^{1}$ (where $z$ is the standard coordinate) and the forms $\phi\left(\tilde{E}_{j}\right)$ on $B \times \mathbf{P}^{1}$.

Let

$$
I=\sum_{J \geqq 0}(-1)^{j} \int_{\mathbf{P}^{1}} \phi\left(\tilde{E}_{j}\right) \log |z|^{2} .
$$

This is a form in $P$, and $\tilde{\phi}(E)$ will be the class of $-I$ modulo $P^{\prime}$.

To prove i), note that the differentials $B \times \mathbf{P}^{1}$ decompose as $\partial=\partial^{B}+\partial_{z}$ and $\bar{\partial}=\bar{\partial}^{B}+\bar{\partial}_{z}$. Since $\partial\left(\phi\left(\widetilde{E}_{j}\right)\right)=\bar{\partial}\left(\phi\left(\widetilde{E}_{j}\right)\right)=0$, we get

$$
\partial^{B} \bar{\partial}^{B}(I)=\sum_{j=0}^{m}(-1)^{j} \int_{\mathbf{P}^{1}} \partial^{B} \bar{\partial}^{B}\left(\phi\left(\tilde{E}_{j}\right)\right) \log |z|^{2}=\sum_{j=0}^{m}(-1)^{j} \int_{\mathbf{P}^{1}} \partial_{z} \bar{\partial}_{z}\left(\phi\left(\tilde{E}_{j}\right)\right) \log |z|^{2} .
$$

Let $\delta_{z}$ be the Dirac mass at $z$. By Stokes formula and the current equation

$$
(-1 / 2 \pi i) \partial_{z} \bar{\partial}_{z} \log |z|^{2}=\delta_{0}-\delta_{\infty},
$$

we get

$$
\partial^{B} \bar{\partial}^{B}(I)=\sum_{j=0}^{m}(-1)^{j} \int_{\mathbf{p}^{1}} \phi\left(\tilde{E}_{j}\right) \partial_{z} \bar{\partial}_{z} \log |z|^{2}=-2 \pi i \sum_{j=0}^{m}(-1)^{j}\left(i_{0}^{*} \phi\left(\tilde{E}_{j}\right)-i_{\infty}^{*} \phi\left(\tilde{E}_{j}\right)\right) .
$$

Since $i_{0}^{*}\left(\tilde{E}_{j}\right)=E_{j}$ and $i_{\infty}^{*}\left(\tilde{E}_{j}\right)=F_{j} \oplus F_{j+1}$ we get i).

To prove ii) we need to prove that the class of $I$ in $P / P^{\prime}$ does not depend on the choice of metrics $\tilde{g}_{j}$ on $\tilde{E}_{j}$ such that the isomorphisms $i_{0}^{*}\left(\tilde{E}_{j}\right) \simeq E_{j}$ and $i_{\infty}^{*}\left(\widetilde{E}_{j}\right) \simeq$ $F_{j} \oplus F_{j+1}$ are isometries.

Let $\tilde{g}_{j}^{\prime}$ be another choice with the same properties. Consider the product $B \times \mathbf{P}^{1} \times \mathbf{P}^{1}$, with points $(y, z, u)$. Define $i_{z}(y, u)=(y, z, u), j_{u}(y, z)=(y, z, u)$, and $p(y, z, u)=(y, z)$. On the bundle $\widetilde{\widetilde{E}}_{j}=p^{*} \widetilde{E}_{j}$, we can choose a metric $\tilde{\tilde{g}}_{j}$ such that $i_{0}^{*}\left(\widetilde{E}_{j}\right)$ is isometric to $p^{*} E_{j}, i_{\infty}^{*}\left(\widetilde{\widetilde{E}}_{j}\right)$ is isometric to $F_{j} \oplus F_{j+1}, j_{0}^{*} \widetilde{\widetilde{E}}_{j}$ is isometric to $\left(\widetilde{E}_{j}, \tilde{g}_{j}\right)$ and $j_{\infty}^{*} \widetilde{\widetilde{E}}_{j}$ is isometric to $\left(\widetilde{E}_{j}, \tilde{g}_{j}^{\prime}\right)$.

Let $\partial=\partial^{B}+\partial_{z}+\partial_{u}$ and $\bar{\partial}=\bar{\partial}^{B}+\bar{\partial}_{z}+\bar{\partial}_{u}$ be the differentials on $B \times \mathbf{P}^{1} \times \mathbf{P}^{1}$. For all $j=0, \ldots, m$ define

$$
\omega_{j}=-\frac{1}{2 \pi i} \int_{\mathbf{P}^{1} \times \mathbf{P}^{1}} \partial_{z} \bar{\partial}_{z}\left(\phi\left(\tilde{\widetilde{E}}_{j}\right)\right) \log |z|^{2} \log |u|^{2} .
$$

By Stokes formula, we get

$$
\begin{aligned}
\omega_{j} & =-\frac{1}{2 \pi i} \int_{\mathbf{P}^{1}} \phi\left(\mathbf{P}^{1}\right. \\
& =\int_{u \in \mathbf{P}^{1}}\left(i_{0}^{*} \phi\left(\tilde{\tilde{E}}_{j}\right)-i_{\infty}^{*} \phi\left(\overline{\tilde{\partial}}_{z} \log |z|^{2} \log |u|^{2}\right) \log |u|^{2}=0,\right.
\end{aligned}
$$

since $i_{0}^{*}\left(\tilde{\widetilde{E}}_{j}\right)$ and $i_{\infty}^{*}\left(\tilde{\widetilde{E}}_{j}\right)$ do not depend on $u$. On the other hand, since $\phi\left(\tilde{\widetilde{E}}_{j}\right)$ is 
$\partial$-closed and $\bar{\partial}$-closed,

$$
\begin{aligned}
\omega_{j} \equiv & -\frac{1}{2 \pi i} \int_{\mathbf{P}^{1} \times \mathbf{P}^{1}} \partial_{u} \bar{\partial}_{u} \phi\left(\tilde{\widetilde{E}}_{j}\right) \log |z|^{2} \log |u|^{2} \\
= & \int_{z \in \mathbf{P}^{1}}\left(j_{0}^{*} \phi\left(\tilde{\widetilde{E}}_{j}\right)-j_{\infty}^{*} \phi\left(\tilde{\widetilde{E}}_{j}\right)\right) \log |z|^{2}=\int_{\mathbf{P}^{1}} \phi\left(\tilde{E}_{j}, \tilde{g}_{j}\right) \log |z|^{2} \\
& -\int_{\mathbf{P}^{1}} \phi\left(\tilde{E}_{j}, \tilde{g}_{j}^{\prime}\right) \log |z|^{2} .
\end{aligned}
$$

This implies that, modulo $P^{\prime}, I$ does not depend on the choice of $\tilde{g}_{j}$. Therefore ii) holds. To prove iii) just notice that $\tilde{g}_{j}$ can be taken independent of $z$ when $E$ is split.

It remains to show that $\widetilde{\phi}(E)$ is uniquely determined by the Properties i), ii) and iii). But assume that a class $\widetilde{\phi}(E)$ has been defined with these properties. Given any acyclic complex $E$ consider the complex $\tilde{E}$ on $B \times \mathbf{P}^{1}$. By property i), we have

$$
(1 / 2 \pi i) \partial \bar{\partial} \tilde{\phi}(\tilde{E})=\sum_{j=0}^{m}(-1)^{j}\left(\phi\left(\tilde{E}_{j}\right)-\phi\left(\tilde{F}_{j} \oplus \tilde{F}_{j+1}\right)\right) .
$$

From the proof of ii) and iii) above we deduce that

$$
\sum_{j=0}^{m}(-1)^{j} \int_{\mathbf{P}^{1}} \phi\left(\tilde{F}_{j} \oplus \tilde{F}_{j+1}\right) \log |z|^{2} \in P^{\prime} .
$$

Therefore

$$
\begin{aligned}
I & =(1 / 2 \pi i) \int_{\mathbf{P}^{1}} \partial \bar{\partial}(\tilde{\phi}(\tilde{E})) \log |z|^{2}=(1 / 2 \pi i) \int_{\mathbf{P}^{1}} \partial_{z} \bar{\partial}_{z}(\tilde{\phi}(\tilde{E})) \log |z|^{2} \\
& =i_{\infty}^{*}(\tilde{\phi}(\tilde{E}))-i_{0}^{*}(\tilde{\phi}(\tilde{E})) .
\end{aligned}
$$

Since $i_{0}^{*}(\widetilde{E})$ and $i_{\infty}^{*}(\widetilde{E})$ is split, we get, by ii) and iii) applied to $\widetilde{\phi}, I \equiv-\tilde{\phi}(E)$.

Let us assume now that $\phi$ is the Chern character ch defined by $\operatorname{ch}(A)=\operatorname{Tr} \exp (A)$ if $A \in M_{k}(\mathbf{C})$. Since $\operatorname{ch}\left(F_{j} \oplus F_{j+1}\right)=\operatorname{ch}\left(F_{j}\right)+\operatorname{ch}\left(F_{j+1}\right)$ we get (see also [GS1], up to a factor of 2)

$$
(1 / 2 \pi i) \partial^{B} \bar{\partial}^{B} \tilde{\operatorname{ch}}(E)=\sum_{j=0}^{m}(-1)^{j} \operatorname{ch}\left(E_{j}\right)
$$

When $g_{0}$ and $g$ are two metrics on a vector bundle $E$ we shall write $\widetilde{c h}\left(g_{0}, g\right)$ instead of $\tilde{c h}\left(\left(E, g_{0}\right) \stackrel{\text { Id }}{\longrightarrow}(E, g)\right)$ (and similarly $\left.\tilde{\phi}\left(g_{0}, g\right)\right)$. If $\alpha \in P$ has degree $(p, p)$, define $\alpha^{*}=(2 \pi i)^{p} \alpha$. By linearity we extend the map $\alpha \mapsto \alpha^{*}$ to any $\alpha \in P$.

\section{Corollary 1.30 .}

i) When $E$ is any acyclic complex as above,

$$
\widetilde{\operatorname{ch}}(E)^{*} \equiv \zeta_{E}^{\prime}(0) .
$$

ii) If $g_{0}$ and $g$ are two metrics on $E$,

$$
\widetilde{\operatorname{ch}}\left(g_{0}, g\right)^{*} \equiv f(g) .
$$

Proof. We just need to show that (up to normalization constants) $\zeta_{E}^{\prime}(0)$ and $f(g)$ 
satisfy the properties i), ii) and iii) characterizing ch. From (1.60) and (1.113) we know that i) is satisfied. The property ii) is clear, and iii) is certainly true for $f(g)$ (i.e. $f\left(g_{0}\right)=0$ ). If $E$ is split the map $v$ commutes with $\nabla$ and $v v^{*}+v^{*} v=\mathrm{Id}$. So we get

$$
\zeta_{E}(s)=-\frac{1}{\Gamma(s)} \int_{0}^{\infty} u^{s-1} e^{-s} d u \operatorname{Tr}_{s}\left(N \exp \left(-\nabla^{2}\right)\right)=-\operatorname{Tr}_{s}\left(N \exp \left(-\nabla^{2}\right)\right)
$$

Therefore $\zeta_{E}^{\prime}(0)=0$ as required.

Remark 1.31. Defining $\zeta_{g_{0}, g}^{\prime}(0)=\zeta_{E}^{\prime}(0)$ with $E=\left(\left(E_{0}, g_{0}\right) \stackrel{\text { Id }}{\longrightarrow}\left(E_{1}, g\right)\right)$ we get

$$
\zeta_{g_{0}, g}^{\prime}(0) \equiv f(g)
$$

and so by the definition of $f(g)$, we find that

$$
\zeta_{g, g^{\prime}}^{\prime}(0)+\zeta_{g^{\prime}, g^{\prime \prime}}^{\prime}(0) \equiv \zeta_{g, g^{\prime \prime}}^{\prime}(0) \text {. }
$$

The equality (1.127) can be shown directly by proving the formula

$$
d^{\mathbf{M}} \zeta_{g_{0}, g}^{\prime}(0) \equiv-\operatorname{Tr}\left(g^{-1} d^{\mathbf{M}} g \exp \left(-\left(\nabla^{g}\right)^{2}\right)\right)
$$

and (1.128) also follows from Theorem 1.22.

In [Bot C, 4.18], Bott and Chern define $\widetilde{c h}(E)$ when $m=2$ and the metrics on $E_{0}$ and $E_{2}$ are induced from the metric on $E_{1}$.

Part ii) of the Corollary extends to arbitrary characteristic classes.

Acknowledgements. We thank J.-B. Bost, S. Donaldson and D. Quillen for helpful discussions. We are also grateful to Mrs Barbichon (Orsay) and Mrs Breiner (IHES) for typing this paper.

\section{References}

[A1] Alvarez, O.: Conformal anomalies and the Index Theorem, to appear in Nucl. Phys.

[AHS] Atiyah, M. F., Hitchin, N. J., Singer, I. M.: Self-duality in four dimensional Riemannian geometry. Proc. Royal Soc. Lond. A 362, 425-461 (1978)

[AS] Atiyah, M. F., Singer, I. M.: The index of elliptic operators IV. Ann. Math. 93, 119-138 (1971)

[BeK] Belavın, A. A., Knizhnik, V. G.: Algebraic geometry and the geometry of quantum strings. Phys. Lett. B 168, 201-206 (1986)

[BF1] Bismut, J.-M., Freed, D. S.: The analysis of elliptic families I, Metrics and connections on determinant bundles. Commun. Math. Phys. 106, 159-176 (1986)

[BF2] Bismut, J.-M., Freed, D. S.: The analysis of elliptic families II, Dirac operators, êta invariants and the holonomy Theorem. Commun. Math. Phys. 107, 103-163 (1986)

[BGS1] Bismut, J.-M., Gillet, H., Soulé, C.: Torsion analytique et fibrés déterminants holomorphes. C. R. Acad. Sci. Paris 305, Série I, $81-84$ (1987)

[BGS2] Bismut, J.-M., Gillet, H., Soulé, C.: Analytic torsion and holomorphic determinant bundles, II: Direct images and Bott-Chern forms. Commun. Math. Phys. 115, 79-126 (1988)

[BGS3] Bismut, J.-M., Gillet, H., Soulé, C.: Analytic torsion and holomorphic determinant bundles, III: Quillen metrics on holomorphic determinants. To appear in Commun. Math. Phys.

[Bo] Bost, J. B.: Conformal and holomorphic anomalies on Riemann surfaces and determinant line bundles. Preprint (1986)

[BotC] Bott, R., Chern, S. S.: Hermitian vector bundles and the equidistribution of the zeros of their holomorphic sections. Acta Math. 114, 71-112 (1968)

[D1] Donaldson, S.: Anti-self-dual Yang-Mills connections over complex algebraic surfaces and stable vector bundles. Proc. Lond. Math. Soc. 50, 1-26 (1985) 
[D2] Donaldson, S.: Infinite determinants, stable bundles and curvature. Preprint 1986

[F] Freed, D.: Determinants, torsion and strings. Commun. Math. Phys. 107, 483-513 (1986)

[GS1] Gillet, H., Soulé, C.: Classes caractéristiques en théorie d'Arakelov. C. R. Acad. Sci. Paris , Série I, 301, 439-442 (1985)

[GS2] Gillet, H., Soulé, C.: Direct images of Hermitian holomorphic bundles. Bull. AMS 15, 209-212 (1986)

[KM] Knudsen, F. F., Mumford, D.: The projectivity of the moduli space of stable curves I: Preliminaries on "det" and "div". Math. Scand. 39, 19-55 (1976)

[P] Polyakov, A. M.: Quantum geometry of bosonic strings. Phys. Lett. B 103, 207-210 (1981)

[Q1] Quillen, D.: Superconnections and the Chern character. Topology 24, 89-95 (1985)

[Q2] Quillen, D.: Determinants of Cauchy-Riemann operators over a Riemann surface. Funct. Anal. Appl. 19, 31-34 (1985)

[RS] Ray, D. B., Singer, I. M.: Analytic torsion for complex manifolds. Ann. Math. 98, 154-177 (1973)

Communicated by A. Jaffe

Received March 30, 1987; in revised form June 16, 1987 